\title{
Top-down enrichment guides in formation of synthetic microbial consortia for biomass degradation
}

\author{
by Gilmore, S.P., Lankiewicz, T.S., Wilken, S.E., Brown, \\ J.L., Sexton, J.A., Henske, J.K., Theodorou, M.K., \\ Valentine, D.L. and O'Malley, M.A
}

Copyright, Publisher and Additional Information: This document is the unedited Author's version of a submitted Work that was subsequently accepted for publication in ACS Synthetic Biology copyright (C) American Chemical Society after peer review. To access the final edited and published work see https://doi.org/10.1021/acssynbio.9b00271

Please refer to any applicable terms of use of the publisher.

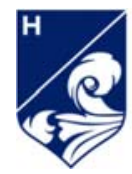


2 Sean P. Gilmore ${ }^{1}$, Jessica A. Sexton¹, John K. Henske, Mike K. Theodorou², David L.

3 Valentine $^{3}$ and Michelle A. O’Malley ${ }^{1^{*}}$

4 1Department of Chemical Engineering, University of California, Santa Barbara, Santa

5 Barbara, CA, 93106, United States

$6 \quad{ }^{2}$ Harper Adams University, Shropshire, United Kingdom

7 3Department of Earth Science, University of California, Santa Barbara, Santa Barbara, CA,

893106, United States

10 Abstract

11 Consortia-based approaches are a promising avenue towards efficient bioprocessing.

12 However, many complex microbial interactions dictate community dynamics and stability.

13 The rumen of large herbivores harbors a network of biomass-degrading fungi and bacteria,

14 as well as archaea and protozoa that work together to degrade lignocellulose, yet the

15 microbial interactions responsible for consortia stability and activity remain

16 uncharacterized. In this work, we demonstrate a novel enrichment-based isolation method

17 selecting for a minimal biomass-degrading community containing anaerobic fungi,

18 methanogenic archaea, and bacteria. The enriched culture displayed an increase of up to 2.1

19 times the growth rate and 1.9 times the fermentation gas produced by the isolated fungus

20 from the enriched culture alone. Metagenomic sequencing revealed functional

21 compartmentalization of the community spread across anaerobic fungi (Piromyces), bacteria

22 (Sphaerochaeta), and methanogens (Methanosphaera and Methanocorpusculum). The

23 minimal consortium enabled more complete degradation of biomass, including 
24 hemicelluloses like xylan and pectin and a wider range of sugar utilization like xylose and 25 galacturonate. Complementing the "top-down" enrichment analysis, a synthetic rumen 26 system was formed from the "bottom-up" with isolated fungi (Piromyces finnis or

27 Neocallimastix californiae) and methanogens (Methanobacterium bryantii) where only the

28 hydrogenotrophic connection was preserved between members. These synthetic consortia

29 also showed improved growth rate and degradation compared to fungi alone, yet lack the

30 temporal stability seen in native consortia, remaining in culture together for fewer than 10

31 transfers on average. Taken together, these two complementary approaches both resulted

32 in productive microbial consortia with faster growth rates and wider substrate uptake than

33 mono-cultured fungi.

34

35 Keywords

36 Microbial Consortia, Anaerobic Fungi, Lignocellulose, Metagenomics, Biomass

37

38 


\section{Introduction}

Artificial, or synthetic, consortia hold the potential to revolutionize bioprocessing,

41 because of their increased efficiency by distributing difficult processes across the individual

42 members. ${ }^{1}$ Consortia are currently utilized in some processes, like anaerobic digestion,

43 where a complex mixture of microbes convert waste into synthesis gas. Typically, these

44 complex communities are isolated or formed from nature, resulting in mixtures of undefined

45 microbes where the members provide some functional or metabolic benefit to the

46 community. However, when consortia are formed by combining individual microbes from

47 the "bottom-up", wieldy microbes compete for the same resources often resulting in the

48 dominance of one microbe that outperforms the others. Identifying key factors that both

49 connect and stabilize the consortium is critical to overcome current limitations inherent in

50 using microbial communities for bioprocessing.

51 Diverse microbial communities from nature participate in many different types of

52 interactions, ranging from favorable to benign and even unfavorable, which can be leveraged

53 to enhance the productivity of synthetic consortia. ${ }^{2}$ These interactions help regulate

54 community dynamics, resulting in stable macroscopic traits. Syntrophy is one possible

55 interaction that helps tie together microorganisms that have a mutual benefit. ${ }^{3}$ Syntrophy

56 occurs when one microbe utilizes metabolites produced by a second microbe, resulting in an

57 interwoven and dependent metabolism ${ }^{4}$. Obligate syntrophy has further benefits, where the

58 removal of metabolic products allows utilization of substrates by a second microbe that they

59 are unable to catabolize in isolation ${ }^{4}$.

60

One route to identify mechanisms that regulate community formation, diversity, and

61 stability is to investigate "minimal" native consortia, whereby key microbial players have 
62 been enriched from a complex ecosystem. An ecosystem of key biotech relevance is the

63 hindgut of large herbivores, where biomass is routinely degraded and anaerobic gut fungi

64 are primarily responsible for degradation ${ }^{5}$. Though gut fungi have recently been

65 characterized in isolation ${ }^{5,6}$, such growth does not accurately mimic their native

66 environment. Alongside anaerobic fungi, archaea, bacteria, and protozoa are found in

67 herbivore hindguts. Among these, anaerobic fungi have been shown to interact closely with

68 methane producing archaea (methanogens) ${ }^{7}$. Methanogens siphon hydrogen and other

69 metabolites from the fungi, allowing the fungi to more efficiently produce energy by

70 increasing the flux through their hydrogenosomes ${ }^{7}$. In addition to greatly increasing biomass

71 degradation, fungi-methanogen co-cultures are capable of directly converting crude biomass

72 into methane ${ }^{7}$. Early and recent characterization of fungi-methanogen co-cultures ${ }^{7-13}$

73 focused mainly on macroscopic effects of co-culture. Separate studies have considered native

74 consortia - isolated fungi with natively associated methanogens, and in vitro consortia -

75 separately isolated anaerobic fungi and methanogens. No previous studies have made direct

76 comparisons between the two types of cultures, however, so the difference in effectiveness

77 between the two types of cultures are not known.

78 In this study, we utilize "top-down" isolation to inform "bottom-up" reconstruction of

79 a biomass-degrading microbial consortium. First, using next generation sequencing

80 techniques, we identify the interactions between microbes in a minimal consortium isolated

81 from nature. We then demonstrate that the mechanisms identified in the native community

82 can be used to design a synthetic rumen consortium that greatly exceeds the performance of

83 isolated members. The imparted robustness and stability are crucial to developing consortia

84 capable of routine use in waste-to-chemical production. 


\section{Results and Discussion}

87 Selective Enrichment Enables Isolation of a Stable Native Consortium

In order to capture a consortium representative of the rumen microbiome, a selective

89 enrichment process was developed to isolate a minimal system (Figure 1). Methanogens and

90 anaerobic fungi were successfully enriched for, as determined by periodic sampling of

91 methane in the headspace of cultures (Figure S1) and methanogen-specific 16S and fungal

92 ITS primers (Table S1). A rough estimate of diversity was calculated by cloning the ITS or

93 16S amplicons into vectors and selecting individual colonies for Sanger Sequencing. The ITS

94 sequences were highly similar, with only a few slight differences likely due to the difficulty

95 of sequencing ITS regions from anaerobic fungi due to extremely high AT content ${ }^{14}$. The

96 closest cultured match to the enriched fungus was a Piromyces isolate, but with only $86 \%$

97 identity matching a cultured isolate across the entire ITS and 5.8S region. The sequences

98 clustered distinctly from other fungal genera (Figure S2a), grouping into a clade with an

99 uncultured isolate. Microscopy revealed the formation of monocentric sporangia (Figure S3)

100 similar to the genus Piromyces. Methanogen-specific primers amplified two distinct

101 sequences most similar to Methanosphaera and Methanocorpusculum isolates. The

102 phylogenetic identity (Figure S2b) of the two methanogens was somewhat surprising, as the

103 rumen and intestinal tracts of herbivores are often dominated by Methanobrevibacter

104 methanogens ${ }^{15}$. However, it is likely that specific fungal-methanogen interactions led to

105 isolation of the Methanosphaera and Methanocorpusculum over the more abundant 106 Methanobrevibacter. 
108 of the consortium, but was not done for the eukaryotic population due to the complexity in 109 sequencing fungal genomes.6,16 DNA was isolated from the prokaryotic culture after one 110 generation of growth to capture abundances as close to the native community as possible. A 111 summary of the metagenomic sequencing is detailed in Table S2. An initial search for 16S 112 sequences revealed the two methanogens previously detected by methanogen primers, but 113 also a third unique sequence, most similar to a Sphaerochaeta genome. The phylogenetic 114 placement of each $16 \mathrm{~S}$ sequence is shown in Figure S3. The assembled contigs were binned 115 and checked for completion. Each bin was at least 93\% complete with less than 2\% 116 contamination (Figure S4), which qualifies each as a high-quality draft according to the 117 Minimum Information about a Metagenome Assembled Genome ${ }^{17}$.

To compare the growth characteristics of the enriched consortium versus individual 122 microbial constituents, a novel separation scheme was designed to separate the fungal 123 component of the consortium from the prokaryotic part, as shown in Figure 1. While 124 methanogens are resistant to penicillin and streptomycin, they are susceptible to 125 chloramphenicol treatment. As such, pure fungal cultures were obtained by treatment with 126 chloramphenicol. Similarly, fungi require a carbohydrate substrate, and removing that 127 substrate source selected against fungal growth. The culture of fungi, bacteria and 128 methanogens was tested for growth on a wide variety of substrates ranging from simple 129 sugars to complex lignocellulose, and compared to each individual monoculture. Because of 
130 the unique growth habits of anaerobic fungi, total gas production was measured as a proxy

131 for growth, as in previous work ${ }^{18}$.

132 The net specific growth rate across a variety of substrates, calculated from

133 fermentation gas production, is shown in Figure 2A. The mixed culture outperformed either

134 monoculture significantly $(\mathrm{p}<0.05)$ across all substrates except for switch grass. The growth

135 rate of the consortium was up to 2.1 times faster than the isolated fungus alone. The amount

136 of fermentation gas produced was enhanced as well (Figure 2B), where the consortium

137 produced up to 1.9 times more gas than the isolated fungus. Finally, the degradation of

138 substrates was dramatically enhanced, as shown by the visible degradation of filter paper in

139 Figure 2C. The consortium fully disrupted the paper within 68 hours of inoculation, up to

$14040 \%$ faster than the fungus alone. Of the more complex substrates (reed canary grass, alfalfa

141 stem, corn stover, and switch grass) the growth rate was greatest on reed canary grass for

142 both the consortium and isolated fungus. Since the community was isolated using reed

143 canary grass as the substrate, it is likely that the community members were tuned for

144 optimal growth on reed canary grass. Interestingly, there were some substrates, such as

145 xylan and pectin, on which the fungal monoculture had minimal growth, whereas the mixed

146 culture produced significant growth and fermentation gas. No significant growth was

147 demonstrated by the isolated methanogen/bacteria part on any substrate.

150 Metagenomic Analysis Suggests that Syntrophy and Compartmentalized Metabolism Drive 151 Stability 
153 metabolism, despite some redundant capabilities (Figure 3). Syntrophy occurs between the

154 methanogens and fungi, as well as the methanogens and the bacteria via hydrogen exchange

155 and assimilation. However, this is just one part of the compartmentalization observed. From

156 previous work ${ }^{5,6}$, it is known that the anaerobic fungi are enriched in biomass degrading

157 enzymes, but these enzymes are primarily exo- and endo- cellulases. They contain some

158 enzymes for degradation of smaller cellodextran fragments, yet they also have transporters

159 capable of taking up these longer cellulose fragments ${ }^{19}$. In the enriched consortium, the fungi

160 act as the primary degraders of the plant biomass, taking up primarily glucose. When grown

161 in isolation, there are excess sugars present ${ }^{20}$, which allows for the presence of "sugar

162 cheaters" often seen in biomass-degrading consortia 21.

164 few enzymes that act on crystalline cellulose, however, the analysis showed many enzymes

165 that further degrade the small fragments released by the fungal enzymes. The Sphaerochaeta

166 member also contained many enzymes for pectin degradation, which explains the enhanced

167 growth on pectin seen by the mixed culture (Figure 2). The role of Spirochaetes in pectin

168 degradation was recently hypothesized from a moose rumen metagenomic survey ${ }^{22}$, lending

169 further evidence to its role in the rumen consortium. Furthermore, analysis of the

170 transporters and metabolism in Sphaerochaeta showed that it is capable of taking up and

171 utilizing a wide array of sugars like arabinose, mannose, galactose, xylose, and galacturonate

172 (Supplementary Database 2).

173 The two methanogens had similar roles in the consortium, as both act as the terminal

174 electron acceptors in the anaerobic community. However, analysis of the 
175 Methanocorpusculum member revealed a pathway for formate utilization, allowing for 176 growth on either $\mathrm{H}_{2} / \mathrm{CO}_{2}$ or formate. Methanosphaera have previously been shown to require 177 a combination of $\mathrm{H}_{2}$ and methanol for growth, however the genes were present for growth 178 on $\mathrm{H}_{2} / \mathrm{CO}_{2}$ as previously shown for a different Methanosphaera species ${ }^{23}$. Methanol is 179 released during the degradation of pectin and other hemicelluloses ${ }^{24,25}$, which could explain 180 the role of the Methanosphaera. Previous analyses have demonstrated the importance of 181 compartmentalized nitrogen metabolism ${ }^{3}$, however our analysis revealed that 182 compartmentalized carbon metabolism is likely the main factor for stability in the enriched 183 community.

Since the enriched consortium was continuously passaged every 3-4 days, we used $18516 \mathrm{~S}$ and ITS metagenomic profiling 5 months after shotgun metagenomics sequencing to 186 assay stability of consortium membership. The relative abundance of methanogens and 187 bacteria was calculated from the metagenomics data by counting the number of reads 188 aligned to each genome bin. As shown in Figure 4, the composition of the consortium and the 189 ratio amongst the prokaryotic members was relatively stable across both time points 190 sampled. In addition, the ITS sampling confirmed that the chloramphenicol-treated fungal 191 monoculture had the same ITS sequence as the enriched culture (Table S1), suggesting that 192 chloramphenicol treatment had no effect on the fungus present. Important to note, however, 193 was that the rumen fluid in the culture medium produced a background $16 \mathrm{~S}$ signal, even 194 though clarified rumen fluid was used. The analysis, therefore, only contained sequences 195 significantly enriched compared to the background uncultured medium control, and it is 196 possible the signal of very low abundance members was masked by the background DNA 197 from clarified rumen fluid. 
"Synthetic Rumen" Systems Drive Enhanced Fermentation \& New Growth Phenotypes

Given the compartmentalization revealed in the minimal rumen consortium, we

201 formed synthetic consortia composed of microbes with similar metabolic dependencies and

202 tested for biomass-degrading activity and stability in batch culture. Piromyces finnis and

203 Neocallimastix californiae are well-studied members of the Neocallimastigomycota with

204 high-quality genomes ${ }^{6}$, and both were paired separately with methanogen

205 Methanobacterium bryantii in culture. As shown in Figure 5, the synthetic co-cultures

206 showed much greater total fermentation gas production (2.1x for P. finnis and 1.8x for $N$.

207 californiae) compared to monocultures of fungi alone on simple biopolymers like Avicel and

208 xylan. However, the synthetic co-cultures produced less total fermentation gas from reed

209 canary grass than the native consortium, and did not show an improvement over the

210 individual fungi alone. Despite the decreased gas production on reed canary grass, it is likely

211 that the co-cultures metabolized more total carbon than the isolated fungi alone.

212 Methanogenesis by $M$. bryantii consumes 5 moles of gas $\left(4 \mathrm{H}_{2}+1 \mathrm{CO}_{2}\right)$ for every 1 mole of

213 methane produced, and methane was at a concentration of $\sim 10 \%$ at the end of growth

214 (Figure 5B), suggesting that the total gas produced by the fungi in co-culture was greater

215 than that of either fungus alone.

216 Interestingly, although P. finnis has the necessary genes for metabolism of xylose (and

217 xylan $)^{6}$, it is incapable of utilizing it as the sole growth source under medium conditions used

218 in this study (Figure 5C). However, the synthetic P. finnis-M. bryantii co-culture grew almost

219 as well on xylan as on the glucose-based Avicel. The reason for the recovered phenotype of

220 xylan utilization is unknown, however it is an important observation due to the high 
221 proportion of xylose in biomass. Xylose utilization by members of the Piromyces genus has

222 received attention in recent years as they possess a xylose isomerase instead of xylose

223 reductase/xylitol dehydrogenase typical of other fungi ${ }^{26}$. The xylose isomerase pathway is

224 more commonly found in bacteria, and might have arisen through horizontal gene transfer

225 as many of the cellulases of anaerobic fungi have ${ }^{6}$, which could explain the difficulty in

226 metabolizing pure xylose or xylan. Another hypothesis is related to the availability of

227 required metal cofactors for the xylose isomerase. The xylose isomerase from closely-related

228 Piromyces sp. E2 was shown to operate optimally with a $\mathrm{Mn}^{2+}$ cofactor in place of other

229 bivalent metal ions ${ }^{27,28}$. M. bryantii requires several bivalent cofactors ${ }^{23}$ and therefore likely

230 affects the availability of these molecules to $P$. finnis in co-culture, either making $\mathrm{Mn}^{2+}$ more

231 available or depleting the availability of $\mathrm{Fe}^{2+}$ or $\mathrm{Mg}^{2+}$.

232 One major limitation of the synthetic co-cultures was stability. Although the synthetic

233 rumen systems of $M$. bryantii paired with either P. finnis or $N$. californiae were cultivatable

234 for several generations, they never reached the $>2$ years of stability achieved by the native

235 community, often losing the associated methanogens within a month (10 culture transfers)

236 of formation. Methanogens typically grow much more slowly than anaerobic fungi ${ }^{20,29}$, such

237 that the paired methanogens may be lost due to dilution from consecutive culture transfer.

238 Alternatively, the oxygen sensitivity of methanogens might lead to culture instability through

239 brief oxygen exposure during transfer, largely avoided through potential oxygen scrubbing

240 of the Sphaerochaeta bacterium in the native consortium. The stability seen from the

241 synthetic co-cultures is still an improvement over consortia formed between competing

242 microbes, where competition for resources leads to consortia instability. Importantly, the

243 co-culture requires no genetic engineering like methods such as synthetic signaling ${ }^{30}$ or 
244 engineered auxotrophy ${ }^{1}$ and therefore was applied effectively to microbes lacking genetic 245 tools.

247 Conclusions

250 communities. In both cases, the microbial communities achieved increased fermentation gas

251 compared to the biomass-degrading action of the anaerobic fungal component, and they

252 were capable of utilizing a wider range of substrates. While both top-down and bottom-up

253 methods resulted in consortia that produced more fermentation gas than isolated fungi

254 alone, the top-down approach resulted in a more stable and more productive consortium.

255 This is likely because the microbes present co-evolved in nature and naturally developed

256 mechanisms for stability like syntrophy and compartmentalized carbon metabolism.

257 Starting from a rich pool of microbes in the fecal material of a horse, the top-down approach

258 enriched a mixture of microbes capable of effectively utilizing unpretreated biomass. The

259 methanogens present in the native community are lower abundance members of the rumen,

260 which suggests that other factors selected for their presence in culture over more abundant

261 methanogens. These factors likely contributed to the increased stability of the native

262 community ( $>2$ years) compared to separately isolated methanogen and fungi in the 263 synthetic rumen system ( $\sim 1$ month).

264 There are some limitations of the top-down approach, however. It is dependent on 265 the starting microbial pool and the selective factors used to enrich for the community. The 266 growth substrate utilized likely impacted the enriched community, and even though 
267 anaerobic fungi are among the most robust organisms at degrading biomass, they can be 268 outcompeted during the initial isolation unless antibiotics are present, limiting the total 269 microbial diversity that can be captured. The approach worked well for this application, 270 where the main goal was the utilization of biomass, however the bottom-up approach is 271 more favorable for specific chemical production, or for drop-in modules to create a wider 272 range of products. Future work includes determining the difference in community 273 composition enriched for by changing the selective pressures like growth substrate or 274 antibiotic treatment.

275 In summary, our work displays a direct comparison between natural and synthetic 276 anaerobic consortia for lignocellulosic biomass degradation. The top-down approach 277 resulted in a more productive microbial community and identified compartmentalized 278 carbon metabolism as the main mechanism enabling productivity and stability. The bottom279 up approach allowed for inclusion of microbes with desired properties - in this case 280 sequenced genomes and well-characterized phenotypes. Taken together, these two 281 complementary approaches represent unique paths towards implementing microbial 282 consortia for bioconversion of lignocellulosic biomass. 
Growth Medium, Isolation, and Microbial Cultivation

286 Fungi and consortia were grown in anaerobic fungal Medium C as previously described ${ }^{5}$, 287 supplemented with penicillin $(1000 \mathrm{U} / \mathrm{mL})$, streptomycin $(1000 \mathrm{U} / \mathrm{mL})$, nickel sulfate $(0.2$ $288 \mu \mathrm{g} / \mathrm{mL})$, and sodium 2-mercaptoethanesulfonate $(40 \mu \mathrm{g} / \mathrm{mL})$. Cultures were grown on $1 \%$ 289 (w/v) reed canary grass unless otherwise stated. Avicel (Sigma Aldrich), Xylan (from corn 290 stover, TCI Chemicals, Portland, OR), Pectin (from citrus fruits, MP biomedicals), Reed canary 291 grass, corn stover, switchgrass, and alfalfa stems (all grass obtained from USDA-ARS 292 Research Center, Madison, WI) were added prior to autoclaving media at 1\% (w/v). Glucose 293 and cellobiose were dissolved in water and sterile-filtered then added to media post 294 autoclaving at $0.5 \%(\mathrm{w} / \mathrm{v})$. Methanogens were cultured as previously described 23 , in M2 295 Medium with 80\%/20\% $\mathrm{H}_{2} / \mathrm{CO}_{2}$ headspace, supplemented with methanol (1\% v/v). All 296 cultures were grown at $39^{\circ} \mathrm{C}$ without shaking.

298 Equine fecal materials were collected from the UCSB West Campus Stables, suspended in 299 Medium C, serially diluted, and inoculated into Medium C with reed canary grass 300 supplemented with penicillin, streptomycin, nickel sulfate, and coenzyme M (PS+). Cultures 301 positive for fungal growth were passaged in consecutive batch culture every 3 to 4 days in 302 Medium C supplemented with PS+. The presence of methanogens was verified by periodic 303 determination of methane in the headspace of cultures (Figure S1). In addition, methanogen304 specific $16 \mathrm{~S}^{31}$ and fungal ITS 32 primers were used to confirm the presence of both members 305 (Table S1). A rough estimate of diversity was achieved by cloning the ITS or 16S amplicons 306 into vectors and selecting 10 individual colonies for Sanger Sequencing. 
309 To isolate genomic DNA, cultures were grown in $40 \mathrm{~mL}$ of media in $60 \mathrm{~mL}$ Wheaton serum 310 bottles until stationary phase $\left(\mathrm{OD}_{600} \sim 0.2-0.5\right)$ and then harvested by centrifugation for $31130 \mathrm{~min}$ at $10,000 \times \mathrm{g}$ at $4{ }^{\circ} \mathrm{C}$. Cell pellets were resuspended in $0.5 \mathrm{~mL}$ TE Buffer $(10 \mathrm{mM}$ Tris, 3121 mM EDTA, pH 8.0). Sodium dodecyl sulfate was added to a final concentration of $0.5 \%$, 313 proteinase K (New England BioLabs, Ipswitch, MA) was added to $100 \mu \mathrm{g} / \mathrm{mL}$, and RNaseA 314 (MoBio Laboratories, Carlsbad, CA) was added to $100 \mu \mathrm{g} / \mathrm{mL}$. The mixture was incubated at $31537^{\circ} \mathrm{C}$ for $1 \mathrm{~h}$. $\mathrm{NaCl}$ was added to $0.5 \mathrm{M}$, and $0.5 \mathrm{~mL}$ of phenol:chloroform:isoamyl alcohol 316 (25:24:1) was added. The solution was mixed and then centrifuged at 13,000×g for 10 min 317 at $4{ }^{\circ} \mathrm{C}$. The aqueous phase was transferred to a new tube and $0.6 \mathrm{~mL}$ of isopropyl alcohol 318 was added. The mixture was incubated at $-20{ }^{\circ} \mathrm{C}$ for $\sim 16 \mathrm{~h}$ and then centrifuged at $13,000 \times \mathrm{g}$ 319 for $5 \mathrm{~min}$ at $4{ }^{\circ} \mathrm{C}$. The pellet was washed with $70 \%$ ethanol, centrifuged at 13,000×g for 5 min 320 at $4{ }^{\circ} \mathrm{C}$, and finally resuspended in $10 \mathrm{mM}$ Tris buffer $\mathrm{pH} 8.0$ and stored at $-20{ }^{\circ} \mathrm{C} 33$.

322 Genomic DNA (gDNA) was prepared for high throughput sequencing (HTS) using the TruSeq 323 DNA PCR-Free library prep kit supplied by Illumina, Inc. (San Diego, CA). Briefly, purified 324 gDNA were first fragmented using a Covaris (Woburn, Massachusetts) M220 Focused 325 Ultrasonicator, followed by end repairs, size selection ( $\sim 330 \mathrm{bp})$, end adenylation and 326 paired-end adapters ligation using the kit. Prepped libraries were then quantified using 327 Qubit (Life Technologies, Carlsbad, CA) and TapeStation (Agilent, Santa Clara, CA), before 328 pooling. HTS was performed with an Illumina NextSeq500 sequencer using a 150 cycle, mid 329 output kit (2x75 paired-end). 
Metagenomic Binning and Analysis

332 Metagenomic reads were assembled using Megahit v1.1.2 ${ }^{34}$. Assembled contigs were binned 333 using MetaBAT v2.12.1 35 and CONCOCT v0.4.1 36, with BLAST used to manually curate 334 unbinned contigs. Binned genomes were annotated with the Department of Energy Systems 335 Biology Knowledgebase (KBase, http://kbase.us) automated pipeline. Genomic features 336 including ORFs, large repeat regions, rRNAs, CRISPRs, and tRNAs were identified and 337 annotated with the Rapid Annotations using Subsystems Technology toolkit (RASTtk) ${ }^{37}$. 338 These gene annotations were combined with biochemical information from the Kyoto 339 Encyclopedia of Genes and Genomes (KEGG) ${ }^{38}$ to reconstruct the metabolism of each genome 340 bin. Genome completion was determined utilizing CheckM v1.0.7 39. Metagenomic 341 abundance for each bin was calculated by mapping reads to the full assembly using Bowtie2 342 v2.3.2 40. Transporters were classified using the Transporter Classification DataBase ${ }^{41}$, 343 downloaded on January 15, 2015. Results were filtered to only include hits that covered 70\% 344 of both query and subject with an E-value less than $10^{-3}$. Hits were then manually curated to 345 classify the most likely sugar specificity. CAZymes were predicted using dbCAN ${ }^{42} \mathrm{v}^{4}$ accessed 346 January 29, 2016.

$348 \quad 16 S \&$ ITS Profiling

349 For ribosomal profiling, cultures were grown for 4 days and harvested by centrifugation at $3503220 \mathrm{x}$ g for 20 minutes at $4^{\circ} \mathrm{C}$. DNA was extracted using the FastDNA SPIN kit for soil (MP 351 Biomedicals, Santa Ana, CA, USA) according to the instructions. 16S Primers were designed 352 using the Ribosomal Database Project ${ }^{43}$ targeting the V5 region. ITS primers were as 
353 previously described ${ }^{16}$. Primers had overhangs compatible with Nextera XT primers (P5 for

354 forward and P7 for reverse). The sequences of all primers used are in Table S1. Amplification 355 was performed in $50 \mu \mathrm{L}$ reactions composed of $1 \mu \mathrm{L}$ of extracted DNA, $10 \mu \mathrm{L}$ of 5X Phusion 356 GC Buffer, $1 \mu \mathrm{L}$ of $10 \mathrm{mM}$ dNTPs, $2.5 \mu \mathrm{L}$ of $10 \mu \mathrm{M}$ forward primer, $2.5 \mu \mathrm{L}$ of $10 \mu \mathrm{M}$ reverse 357 primer, $0.5 \mu \mathrm{L}$ of Phusion DNA Polymerase (New England BioLabs, Ipswitch, MA), and 32.5 $358 \mu \mathrm{L}$ of DNase-free $\mathrm{H}_{2} \mathrm{O}$. Amplification occurred with an initial 30 second denaturation at $98^{\circ} \mathrm{C}$; 359 followed by 30 cycles of 10 seconds at $98^{\circ} \mathrm{C}, 30$ seconds at $57^{\circ} \mathrm{C}$, and 30 seconds at $72^{\circ} \mathrm{C}$; a 360 final extension of 5 minutes at $72^{\circ} \mathrm{C}$; and a hold at $4^{\circ} \mathrm{C}$. Prepped libraries were then 361 quantified using Qubit (Life Technologies, Carlsbad, CA) and TapeStation (Agilent, Santa 362 Clara, CA), before pooling. HTS was performed with an Illumina NextSeq500 sequencer using 363 a 150 cycle, mid output kit (2x75 paired-end). Both $16 \mathrm{~S}$ and ITS reads were analyzed using 364 QIIME $^{44}$ version 1.9.1. OTUs were picked using UCLUST ${ }^{45}$ version 1.2.22q. The Greengenes ${ }^{46}$ 365 database version 13.8 was used to classify $16 \mathrm{~S}$ reads, and the UNITE $^{47}$ database version 7 366 was used to classify ITS reads.

Methane Detection

369 Methane concentration was measured with a Shimadzu GC 14A equipped with an N-octane 370 on Res-Sil C column at $50^{\circ} \mathrm{C}$ with $\mathrm{N}_{2}$ carrier gas. Methane standards were run prior to sample 371 analysis and results used to generate a standard curve. Standards were 10, 500, 2500, $37210,000(1 \%), 25,000(2.5 \%)$, and 100,000 (10\%) ppm methane. Range was typically $10^{1}$, 373 with $10^{2}$ for high methane samples. Methane ppm for each test sample was found by 374 comparing against the standard curve. 
377 Raw reads from whole genome metagenomic sequencing and from amplicon metagenomic 378 sequencing have been deposited under the accession number PRJNA471522.

\section{Supporting Information.}

381 Table S1: Measured 16S and ITS sequences

382 Table S2: Metagenomic sequencing statistics

383 Table S3: Binned genome statistics

384 Figure S1: Methane Production over time

385 Figure S2: Phylogeny of Consortia Members

386 Figure S3: Morphological features of Piromyces sp. H1B2

387 Figure S4: Genome Completion by CheckM

388 Supplemental Database 1: CAZyme analysis of metagenomes

389 Supplemental Database 2: Transporter analysis of metagenomes

\section{Abbreviations}

392 ITS - Internal Transcribed Spacer, CAZyme - Carbohydrate Active enzyme

\section{Author Contributions}

394 SPG, JAS, JKH, and MKT carried out enrichment experiments of the native consortium. SPG

395 and JKH performed the sequencing, and SPG conducted the bioinformatic analyses. SPG 396 compared growth measurements of the native and synthetic consortia. SPG, JAS, MKT, DLV, 397 and MAO planned the experiments. SPG and MAO wrote the manuscript.

\section{Acknowledgements}


399 The authors are grateful to funding sources from the Office of Science (BER), U.S. Department

400 of Energy (DE-SC0010352), the National Science Foundation (MCB-1553721), the Institute

401 for Collaborative Biotechnologies through grant W911NF-09-0001 from the U.S. Army

402 Research Office, and the Camille Dreyfus Teacher-Scholar Awards Program. All authors

403 acknowledge support from the California NanoSystems Institute (CNSI) Challenge Grant

404 Program, supported by the University of California, Santa Barbara and the University of

405 California, Office of the President. SPG also acknowledges support from the National Science

406 Foundation Graduate Research Fellowship Program under Grant DGE 1144085. The authors

407 acknowledge the use of the Biological Nanostructures Laboratory within the California

408 NanoSystems Institute, supported by the University of California, Santa Barbara and the

409 University of California, Office of the President. Computational resources were provided by

410 the Center for Scientific Computing at UCSB and NSF Grant CNS-0960316. 


\section{Figure 1: Complementary methods to establish biomass-degrading microbial}

413 consortia. In "Top-Down" isolation, native communities are enriched by selective antibiotic

414 treatment and consecutive culture. Individual constituents of the native culture can be 415 separated by either removing the sugar source for the fungi selecting for methanogens and 416 bacteria, or by treatment with chloramphenicol selecting for fungi. In "Bottom-Up" 417 reconstruction, separately isolated methanogens and fungi are combined in a culture, filling 418 the roles observed from the native consortium.

420 Figure 2: Native consortium exhibits faster growth and faster substrate degradation

421 than fungus isolated from consortium. (A, B) Across all conditions tested, the native 422 consortium displayed a much greater growth rate (A), up to 2.1 times the isolated fungal part 423 alone. The total amount of fermentation gas produced was also greatly increased (B), up to 4241.9 times the amount of the fungus alone. The improvement was much greater on the simple 425 sugars like glucose and cellobiose than on the more complex grasses such as reed canary 426 grass. Interestingly, minimal growth on pectin or xylan was determined for the fungi alone, 427 whereas the consortium easily consumed them. No growth was determined on any substrate 428 for the isolated methanogen part. Error bars represent the standard deviation of three 429 biological replicates. Significance was tested using the Student's t-test, NS represents not 430 significant, $*$ represents $\mathrm{p}<0.05,{ }^{* *}$ represents $\mathrm{p}<0.01$ (C) The enhanced growth and 431 degradation of substrates by the native consortium can be easily seen when grown on filter 432 paper. The consortium rapidly degraded the filter paper, while the fungi alone were just 433 beginning to degrade it at 68 hours of growth. 
435 Figure 3: Metagenomic analysis of the native consortium reveals compartmentalized

436 substrate degradation and metabolism. An overview of substrate metabolism of each

437 member is displayed. The fungi are primary degraders of biopolymers, degrading the long

438 chains of cellulose and hemicellulose. They consume primarily glucose and do not consume

439 many of the other five and six carbon sugars. The bacterium contains genes to degrade

440 shorter cellulose and hemicellulose fragments, as well as genes to metabolize sugars left

441 behind by the fungi. The Methanosphaera consumes methanol liberated from pectin

442 degradation as well as $\mathrm{H}_{2}$. Finally, the Methanocorpusculum consumes $\mathrm{H}_{2}, \mathrm{CO}_{2}$, and formate.

444 Figure 4: Community abundance remains stable despite continuous growth and

445 culture transfer. Relative ratios of the prokaryotic members are displayed, determined 446 from whole genome metagenomics 14 months after isolation and marker gene 447 metagenomics 19 months after isolation. As displayed, the consortium demonstrated 448 remarkable stability, with the relative ratios remaining essentially the same across two 449 measured time points. This reflects the overall stability of the culture despite continuous 450 culture and batch transfer.

452 Figure 5: Synthetic rumen systems produce much more total fermentation gas than

453 isolated fungi on simple substrates. (A) Total accumulated pressure for cultures of 454 Piromyces finnis, and Neocallimastix californiae paired with Methanobacterium bryantii and 455 the native consortium are displayed for growth on Reed canary grass, Avicel, and Xylan. On 456 avicel, the synthetic rumen system greatly outperformed the fungus alone, producing 457 roughly double the amount of gas for both the synthetic and native consortia. (B) The 
458 maximum concentration of methane measured from growth on Reed canary grass is shown 459 for the native consortium and the two synthetic rumen systems. On reed canary grass, 460 accumulated pressure is similar for both the isolated fungus and synthetic rumen system 461 (Figure 5A), although the final concentration of methane in the headspace was $>10 \%$ 462 suggesting that the fungi produced more gas in the synthetic rumen system. (C) The 463 synthetic rumen system of $P$. finnis paired with $M$. bryantii was able to utilize a wider range 464 of substrates including xylan, whereas P. finnis alone demonstrated no growth on xylan. 465 
Figure 1

Top-Down Isolation

Rumen / Fecal Sample

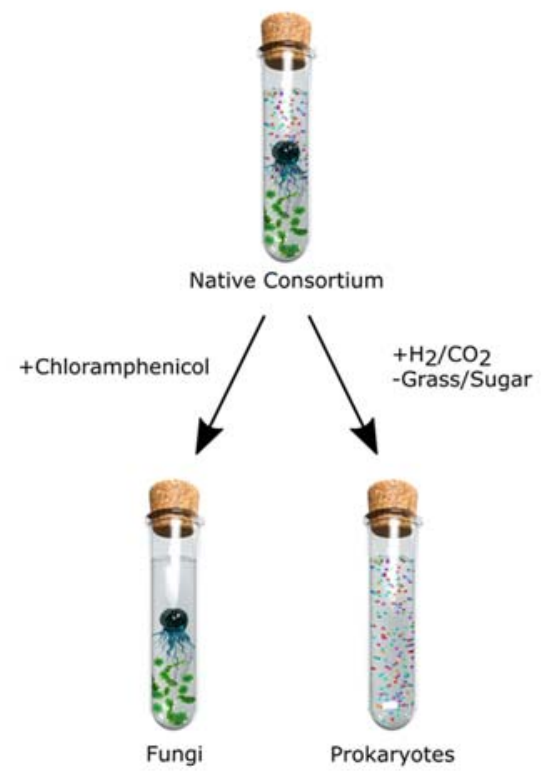

\section{Bottom-Up Reconstruction}

$\begin{array}{ll}\text { Rumen / Fecal Sample } & \begin{array}{l}\text { Rumen / Fecal Sample } \\ \text { Waste Water } \\ \text { Other Environment }\end{array}\end{array}$
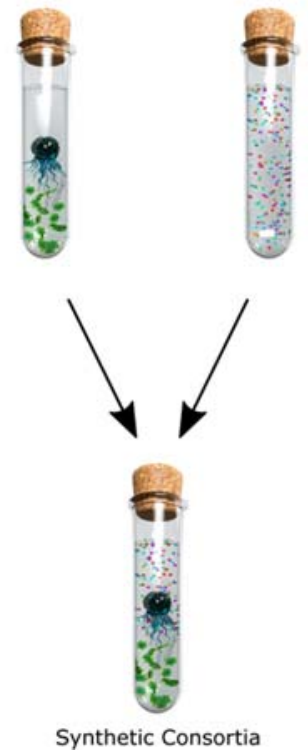
Figure 2

A

469

470
B

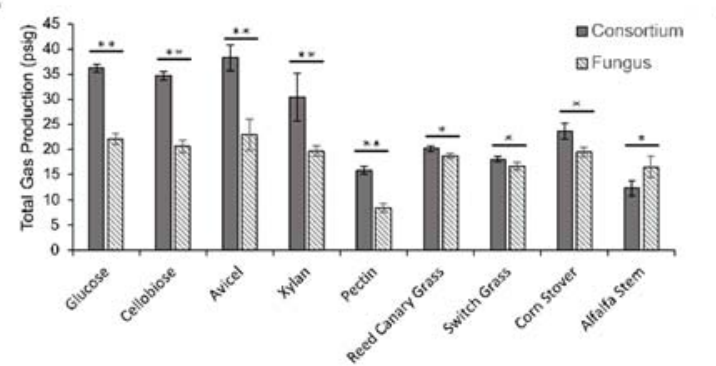

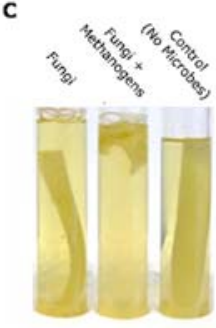

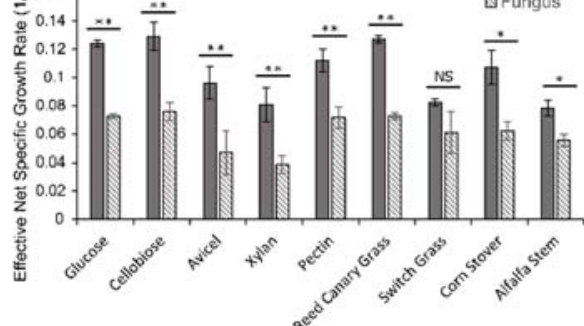


$471 \quad$ Figure 3

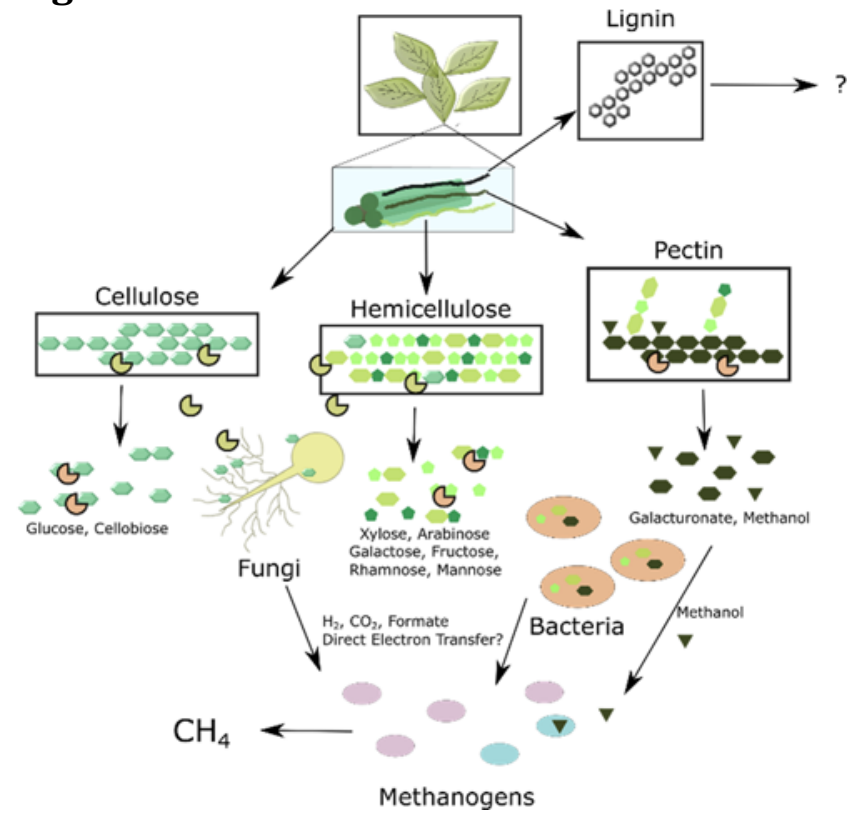

473 
$474 \quad$ Figure 4

Isolation Metagenomics 16S/ITS profiling

475

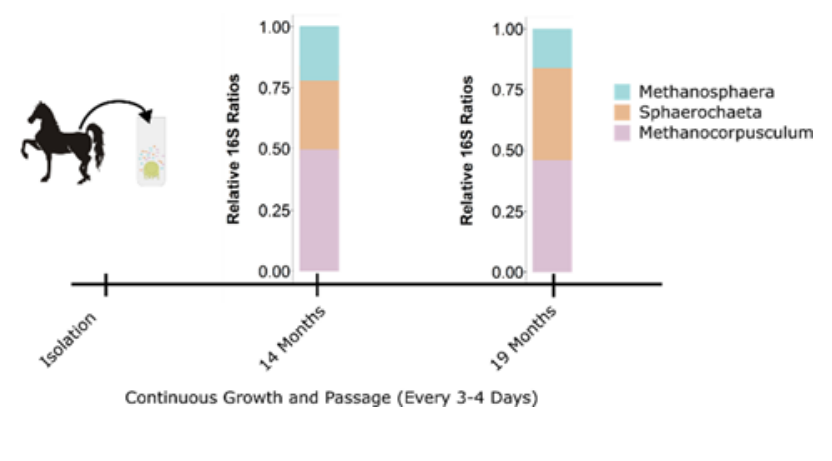


$477 \quad$ Figure 5

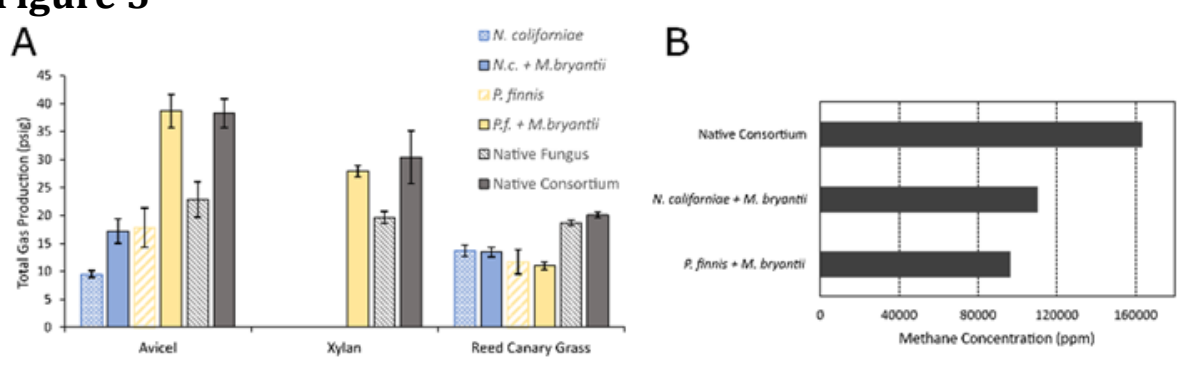

C

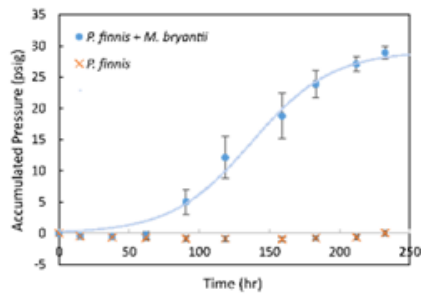




\section{References}

480
[1] Mee, M. T., Collins, J. J., Church, G. M., and Wang, H. H. (2014) Syntrophic exchange in synthetic microbial communities, Proceedings of the National Academy of Sciences 111, E2149-2156.

[2] Peng, X., Gilmore, S., and O'Malley, M. (2016) Microbial communities for bioprocessing: Lessons learned from nature, Current Opinion in Chemical Engineering 14, 103-109.

[3] Embree, M., Liu, J. K., Al-Bassam, M. M., and Zengler, K. (2015) Networks of energetic and metabolic interactions define dynamics in microbial communities, Proceedings of the National Academy of Sciences 112, 15450-15455.

[4] Morris, B. E., Henneberger, R., Huber, H., and Moissl-Eichinger, C. (2013) Microbial syntrophy: interaction for the common good, FEMS Microbiol Rev 37, 384-406.

[5] Solomon, K. V., Haitjema, C. H., Henske, J. K., Gilmore, S. P., Borges-Rivera, D., Lipzen, A., Brewer, H. M., Purvine, S. O., Wright, A. T., Theodorou, M. K., Grigoriev, I. V., Regev, A., Thompson, D. A., and O'Malley, M. A. (2016) Early-branching gut fungi possess a large, comprehensive array of biomass-degrading enzymes, Science 351, 1192-1195.

[6] Haitjema, C. H., Gilmore, S. P., Henske, J. K., Solomon, K. V., de Groot, R., Kuo, A., Mondo, S. J., Salamov, A. A., LaButti, K., Zhao, Z., Chiniquy, J., Barry, K., Brewer, H. M., Purvine, S. O., Wright, A. T., Hainaut, M., Boxma, B., van Alen, T., Hackstein, J. H. P., Henrissat, B., Baker, S. E., Grigoriev, I. V., and O'Malley, M. A. (2017) A parts list for fungal cellulosomes revealed by comparative genomics, Nature Microbiology 2, 17087.

[7] Bauchop, T., and Mountfort, D. O. (1981) Cellulose fermentation by a rumen anaerobic fungus in both the absence and the presence of rumen methanogens, Applied and Environmental Microbiology 42, 1103-1110.

[8] Teunissen, M. J., Kets, E. P. W., Dencamp, H., Intveld, J., and Vogels, G. D. (1992) Effect of coculture of anaerobic fungi isolated from ruminants and nonruminants with methanogenic bacteria on cellulolytic and xylanolytic enzyme-activities, Archives of Microbiology 157, 178182.

[9] Mountfort, D. O., Asher, R. A., and Bauchop, T. (1982) Fermentation of cellulose to methane and carbon dioxide by a rumen anaerobic fungus in a triculture with Methanobrevibacter sp strain RA1 and Methanosarcina barkeri Applied and Environmental Microbiology 44, 128134.

[10] Joblin, K. N., Naylor, G. E., and Williams, A. G. (1990) Effect of Methanobrevibacter smithii on xylanolytic activity of anaerobic ruminal fungi, Applied and Environmental Microbiology 56, 2287-2295.

[11] Joblin, K. N., and Williams, A. G. (1991) Effect of cocultivation of ruminal chytrid fungi with Methanobrevibacter smithii on lucerne stem degradation and extracellular fungal enzymeactivities, Letters in Applied Microbiology 12, 121-124.

[12] Joblin, K. N., Matsui, H., Naylor, G. E., and Ushida, K. (2002) Degradation of fresh ryegrass by methanogenic co-cultures of ruminal fungi grown in the presence or absence of Fibrobacter succinogenes, Current Microbiology 45, 46-53.

[13] Marvinsikkema, F. D., Richardson, A. J., Stewart, C. S., Gottschal, J. C., and Prins, R. A. (1990) Influence of hydrogen-consuming bacteria on cellulose degradation by anaerobic fungi, Applied and Environmental Microbiology 56, 3793-3797.

[14] Edwards, J. E., Forster, R. J., Callaghan, T. M., Dollhofer, V., Dagar, S. S., Cheng, Y., Chang, J., Kittelmann, S., Fliegerova, K., Puniya, A. K., Henske, J. K., Gilmore, S. P., O'Malley, M. A., Griffith, G. W., and Smidt, H. (2017) PCR and Omics Based Techniques to Study the Diversity, Ecology and Biology of Anaerobic Fungi: Insights, Challenges and Opportunities, Frontiers in Microbiology 8, 1657. 
[15] Seedorf, H., Kittelmann, S., and Janssen, P. H. (2015) Few highly abundant operational taxonomic units dominate within rumen methanogenic archaeal species in New Zealand sheep and cattle, Applied Environmental Microbiology 81, 986-995.

[16] Youssef, N. H., Couger, M. B., Struchtemeyer, C. G., Liggenstoffer, A. S., Prade, R. A., Najar, F. Z., Atiyeh, H. K., Wilkins, M. R., and Elshahed, M. S. (2013) The genome of the anaerobic fungus Orpinomyces sp strain C1A reveals the unique evolutionary history of a remarkable plant biomass degrader, Applied and Environmental Microbiology 79, 4620-4634.

[17] Bowers, R. M., Kyrpides, N. C., Stepanauskas, R., Harmon-Smith, M., Doud, D., Reddy, T. B. K., Schulz, F., Jarett, J., Rivers, A. R., Eloe-Fadrosh, E. A., Tringe, S. G., Ivanova, N. N., Copeland, A., Clum, A., Becraft, E. D., Malmstrom, R. R., Birren, B., Podar, M., Bork, P., Weinstock, G. M., Garrity, G. M., Dodsworth, J. A., Yooseph, S., Sutton, G., Glockner, F. O., Gilbert, J. A., Nelson, W. C., Hallam, S. J., Jungbluth, S. P., Ettema, T. J. G., Tighe, S., Konstantinidis, K. T., Liu, W. T., Baker, B. J., Rattei, T., Eisen, J. A., Hedlund, B., McMahon, K. D., Fierer, N., Knight, R., Finn, R., Cochrane, G., Karsch-Mizrachi, I., Tyson, G. W., Rinke, C., Lapidus, A., Meyer, F., Yilmaz, P., Parks, D. H., Eren, A. M., Schriml, L., Banfield, J. F., Hugenholtz, P., and Woyke, T. (2017) Minimum information about a single amplified genome (MISAG) and a metagenomeassembled genome (MIMAG) of bacteria and archaea, Nat Biotechnol 35, 725-731.

[18] Theodorou, M. K., Davies, D. R., Nielsen, B. B., Lawrence, M. I. G., and Trinci, A. P. J. (1995) Determination of growth of anaerobic fungi on soluble and cellulosic substrates using a pressure transducer, Microbiology 141, 671-678.

[19] Seppala, S., Solomon, K. V., Gilmore, S. P., Henske, J. K., and O'Malley, M. A. (2016) Mapping the membrane proteome of anaerobic gut fungi identifies a wealth of carbohydrate binding proteins and transporters, Microb Cell Fact 15, 212.

[20] Henske, J. K., Wilken, S. E., Solomon, K. V., Smallwood, C. R., Shutthanandan, V., Evans, J. E., Theodorou, M. K., and O'Malley, M. A. (2017) Metabolic characterization of anaerobic fungi provides a path forward for bioprocessing of crude lignocellulose, Biotechnology and Bioengineering.

[21] Jimenez, D. J., Dini-Andreote, F., and van Elsas, J. D. (2014) Metataxonomic profiling and prediction of functional behaviour of wheat straw degrading microbial consortia, Biotechnol Biofuels 7, 92.

[22] Svartstrom, O., Alneberg, J., Terrapon, N., Lombard, V., de Bruijn, I., Malmsten, J., Dalin, A. M., El Muller, E., Shah, P., Wilmes, P., Henrissat, B., Aspeborg, H., and Andersson, A. F. (2017) Ninety-nine de novo assembled genomes from the moose (Alces alces) rumen microbiome provide new insights into microbial plant biomass degradation, Isme $j$.

[23] Gilmore, S. P., Henske, J. K., Sexton, J. A., Solomon, K. V., Seppala, S., Yoo, J. I., Huyett, L. M., Pressman, A., Cogan, J. Z., Kivenson, V., Peng, X., Tan, Y., Valentine, D. L., and O'Malley, M. A. (2017) Genomic analysis of methanogenic archaea reveals a shift towards energy conservation, BMC Genomics 18, 639.

[24] Dongowski, G., Lorenz, A., and Anger, H. (2000) Degradation of pectins with different degrees of esterification by Bacteroides thetaiotaomicron isolated from human gut flora, Appl Environ Microbiol 66, 1321-1327.

[25] Ndeh, D., Rogowski, A., Cartmell, A., Luis, A. S., Basle, A., Gray, J., Venditto, I., Briggs, J., Zhang, X., Labourel, A., Terrapon, N., Buffetto, F., Nepogodiev, S., Xiao, Y., Field, R. A., Zhu, Y., O'Neil, M. A., Urbanowicz, B. R., York, W. S., Davies, G. J., Abbott, D. W., Ralet, M. C., Martens, E. C., Henrissat, B., and Gilbert, H. J. (2017) Complex pectin metabolism by gut bacteria reveals novel catalytic functions, Nature 544, 65-70.

[26] Kuyper, M., Harhangi, H. R., Stave, A. K., Winkler, A. A., Jetten, M. S., de Laat, W. T., den Ridder, J. J., Op den Camp, H. J., van Dijken, J. P., and Pronk, J. T. (2003) High-level functional expression of a fungal xylose isomerase: the key to efficient ethanolic fermentation of xylose by Saccharomyces cerevisiae?, FEMS Yeast Res 4, 69-78. 
[27] Verhoeven, M. D., Lee, M., Kamoen, L., van den Broek, M., Janssen, D. B., Daran, J. G., van Maris, A. J., and Pronk, J. T. (2017) Mutations in PMR1 stimulate xylose isomerase activity and anaerobic growth on xylose of engineered Saccharomyces cerevisiae by influencing manganese homeostasis, Nature Scientific Reports 7, 46155.

[28] Lee, M., Rozeboom, H. J., de Waal, P. P., de Jong, R. M., Dudek, H. M., and Janssen, D. B. (2017) Metal Dependence of the Xylose Isomerase from Piromyces sp. E2 Explored by Activity Profiling and Protein Crystallography, Biochemistry 56, 5991-6005.

[29] Godsy, E. M. (1980) Isolation of Methanobacterium bryantii from a deep aquifer by using a novel broth-antibiotic disk method, Applied and Environmental Microbiology 39, 1074-1075.

[30] Marchand, N., and Collins, C. H. (2016) Synthetic Quorum Sensing and Cell-Cell Communication in Gram-Positive Bacillus megaterium, ACS Synth Biol 5, 597-606.

[31] Wright, A. D., and Pimm, C. (2003) Improved strategy for presumptive identification of methanogens using 16S riboprinting, J Microbiol Methods 55, 337-349.

[32] Tuckwell, D. S., Nicholson, M. J., McSweeney, C. S., Theodorou, M. K., and Brookman, J. L. (2005) The rapid assignment of ruminal fungi to presumptive genera using ITS1 and ITS2 RNA secondary structures to produce group-specific fingerprints, Microbiology 151, 1557-1567.

[33] Anderson, I. J., Sieprawska-Lupa, M., Goltsman, E., Lapidus, A., Copeland, A., Del Rio, T. G., Tice, H., Dalin, E., Barry, K., Pitluck, S., Hauser, L., Land, M., Lucas, S., Richardson, P., Whitman, W. B., and Kyrpides, N. C. (2009) Complete genome sequence of Methanocorpusculum labreanum type strain Z, Standards in Genomic Sciences 1, 197-203.

[34] Li, D., Luo, R., Liu, C. M., Leung, C. M., Ting, H. F., Sadakane, K., Yamashita, H., and Lam, T. W. (2016) MEGAHIT v1.0: A fast and scalable metagenome assembler driven by advanced methodologies and community practices, Methods 102, 3-11.

[35] Kang, D. D., Froula, J., Egan, R., and Wang, Z. (2015) MetaBAT, an efficient tool for accurately reconstructing single genomes from complex microbial communities, PeerJ 3, e1165.

[36] Alneberg, J., Bjarnason, B. S., de Bruijn, I., Schirmer, M., Quick, J., Ijaz, U. Z., Lahti, L., Loman, N. J., Andersson, A. F., and Quince, C. (2014) Binning metagenomic contigs by coverage and composition, Nat Methods 11, 1144-1146.

[37] Brettin, T., Davis, J. J., Disz, T., Edwards, R. A., Gerdes, S., Olsen, G. J., Olson, R., Overbeek, R., Parrello, B., Pusch, G. D., Shukla, M., Thomason, J. A., III, Stevens, R., Vonstein, V., Wattam, A. R., and Xia, F. (2015) RASTtk: A modular and extensible implementation of the RAST algorithm for building custom annotation pipelines and annotating batches of genomes, Scientific Reports 5.

[38] Kanehisa, M., Goto, S., Sato, Y., Furumichi, M., and Tanabe, M. (2012) KEGG for integration and interpretation of large-scale molecular data sets, Nucleic Acids Research 40, D109-D114.

[39] Parks, D. H., Imelfort, M., Skennerton, C. T., Hugenholtz, P., and Tyson, G. W. (2015) CheckM: assessing the quality of microbial genomes recovered from isolates, single cells, and metagenomes, Genome Res 25, 1043-1055.

[40] Langmead, B., and Salzberg, S. L. (2012) Fast gapped-read alignment with Bowtie 2, Nat Methods 9, 357-359.

[41] Saier, M. H., Jr., Tran, C. V., and Barabote, R. D. (2006) TCDB: the Transporter Classification Database for membrane transport protein analyses and information, Nucleic Acids Research 34, D181-186.

[42] Yin, Y., Mao, X., Yang, J., Chen, X., Mao, F., and Xu, Y. (2012) dbCAN: a web resource for automated carbohydrate-active enzyme annotation, Nucleic Acids Res 40, W445-451.

[43] Cole, J. R., Wang, Q., Fish, J. A., Chai, B., McGarrell, D. M., Sun, Y., Brown, C. T., Porras-Alfaro, A., Kuske, C. R., and Tiedje, J. M. (2014) Ribosomal Database Project: data and tools for high throughput rRNA analysis, Nucleic Acids Res 42, D633-642.

[44] Caporaso, J. G., Kuczynski, J., Stombaugh, J., Bittinger, K., Bushman, F. D., Costello, E. K., Fierer, N., Pena, A. G., Goodrich, J. K., Gordon, J. I., Huttley, G. A., Kelley, S. T., Knights, D., Koenig, J. E., 
Ley, R. E., Lozupone, C. A., McDonald, D., Muegge, B. D., Pirrung, M., Reeder, J., Sevinsky, J. R., Turnbaugh, P. J., Walters, W. A., Widmann, J., Yatsunenko, T., Zaneveld, J., and Knight, R. (2010) QIIME allows analysis of high-throughput community sequencing data, In Nat Methods, pp 335-336, United States.

[45] Edgar, R. C. (2010) Search and clustering orders of magnitude faster than BLAST, Bioinformatics 26, 2460-2461.

[46] DeSantis, T. Z., Hugenholtz, P., Larsen, N., Rojas, M., Brodie, E. L., Keller, K., Huber, T., Dalevi, D., Hu, P., and Andersen, G. L. (2006) Greengenes, a chimera-checked 16S rRNA gene database and workbench compatible with ARB, Appl Environ Microbiol 72, 5069-5072.

[47] Koljalg, U., Nilsson, R. H., Abarenkov, K., Tedersoo, L., Taylor, A. F., Bahram, M., Bates, S. T., Bruns, T. D., Bengtsson-Palme, J., Callaghan, T. M., Douglas, B., Drenkhan, T., Eberhardt, U., Duenas, M., Grebenc, T., Griffith, G. W., Hartmann, M., Kirk, P. M., Kohout, P., Larsson, E., Lindahl, B. D., Lucking, R., Martin, M. P., Matheny, P. B., Nguyen, N. H., Niskanen, T., Oja, J., Peay, K. G., Peintner, U., Peterson, M., Poldmaa, K., Saag, L., Saar, I., Schussler, A., Scott, J. A., Senes, C., Smith, M. E., Suija, A., Taylor, D. L., Telleria, M. T., Weiss, M., and Larsson, K. H. (2013) Towards a unified paradigm for sequence-based identification of fungi, Mol Ecol 22, 5271-5277.

643

644 
646 Table S1: Primers and Measured 16S and ITS sequences

\begin{tabular}{|c|c|}
\hline $\begin{array}{l}\text { Sanger Sequencing } \\
\text { Methanogen-specific } \\
16 S \text { primers }\end{array}$ & $\begin{array}{l}\text { Met86F: GCTCAGTAACACGTGG } \\
\text { Met1340R: CGGTGTGTGCAAGGAG }\end{array}$ \\
\hline $\begin{array}{l}\text { Methanosphaera sp } \\
\text { H1B2 Sanger } 16 \mathrm{~S} \\
\text { sequence }\end{array}$ & 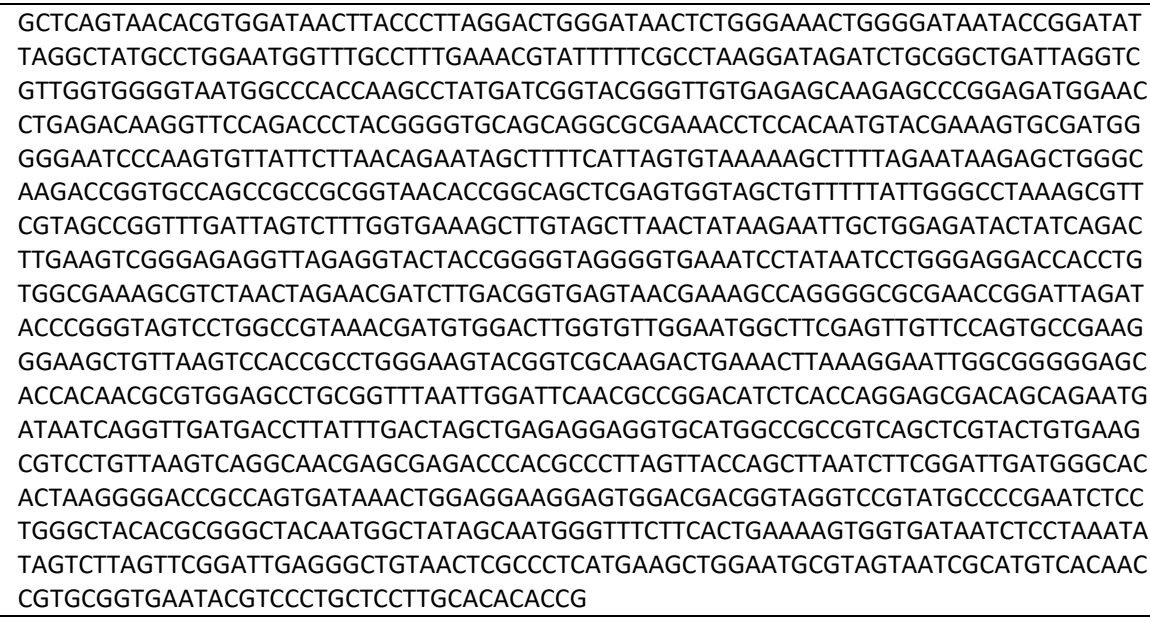 \\
\hline $\begin{array}{l}\text { Methanocorpusculum } \\
\text { sp H1B2 Sanger 16S } \\
\text { sequence }\end{array}$ & $\begin{array}{l}\text { GCTCAGTAACACGTGGTTAATCTGCCCTTGGGTGGAGGATACTCCCGGGAAACTGGGGCTAATACTCCATAG } \\
\text { TGAATGCATGCTGGAATGCTGCATTCTCGAAAGATTCATCGCCCAAGGATGAGACTGCGTCCGATTAGGTCG } \\
\text { TTGGTGGGGTAACGGCCCACCAAGCCTTTTATCGGTACGGGTTGTGGGAGCAAGATCCCGGAGATGGATTCT } \\
\text { GAGACATGAATCCAGGCCCTACGGGGCGCAGCAGGCGCGCAAACTTTACAATGCGAGCAATCGTGATAAGG } \\
\text { AAACCCTGAGTGCCTGTCGATGCAGGCTGTTCATATGTTTAAATTGCATGTGAAGAAAGGGCAGGGCAAGAC } \\
\text { CGGTGCCAGCCGCCGCGGTAATACCGGCTGCTCGAGTGATGGCCACTATTACTGGGTTTAAAGCGTCCGTAG } \\
\text { CTTGACTGTTAGGTCTCTTGGGAAATCTTTTGGCTTAACCAAAAGGCGTCTAAGAGATACCGGCATTCTTGGA } \\
\text { ACTGGGAGAGGTAAGCCGTACTTCGGGGGTAGGAGTGAAATCTGGTAATCCTCGAGGGACGACCTATGGCG } \\
\text { AAGGCAGCTTACCAGAACAGCTTCGACAGTGAGGGACGAAAGCTGGGGAGCAAACGGGATTAGATACCCC } \\
\text { GGTAGTCCCAGCCGTAAACAATGTGCGTTAGGTGTGTCGGTTACCACGCGTAACTGATGCGCCGAAGAGAAA } \\
\text { TCGTGAAACGCACCACCTGGGAAGTACGGTCGCAAGGCTGAAACTTAAAGGAATTGGCGGGGGAGCACCAC } \\
\text { AACAGGTGGAGCCTGCGGTTTAATTGGATTCAACGCCGGACATCTCACCGGATAAGACAGCTGAATGATTGT } \\
\text { CAATCTGAAGGTTTACATGACTAGCTGAGAGGAGGTGCATGGCCGTCGTCAGTTCGTACTGTGAAGCATCC } \\
\text { TGTTAAGTCAGGCAACGAGCGAGACCCACGCCAACAATTGCCAGCAGCATCTCCGGATGGCTGGGGACATTG } \\
\text { TTGGGACCGCCTCTGCTAAAGGGGAGGAAGGAATGGGCAACGGAGGTCAGCATGCCCCGAATTATCCGGG } \\
\text { CTACACGCGGGCTACAATGGCCGGGCAATGGGTAACGACACCGAAAGGTCAGTCAATCTCCTAACCCCGG } \\
\text { CCTTAGTTAGGATTGCGGGTGCAACTCACCCGCATGATCTGGAATCTGTAGTAATCGCGTTCACTATAGC } \\
\text { GCGGTGAATACGTCCCTGCTCCTTGCACACACCG }\end{array}$ \\
\hline $\begin{array}{l}\text { Sanger Sequencing ITS } \\
\text { region primers }\end{array}$ & $\begin{array}{l}\text { JB205: GGAAGTAAAAGTCGTAACAAGG } \\
\text { JB206: TCCTCCGCTTATTAATATGC }\end{array}$ \\
\hline $\begin{array}{l}\text { Piromyces sp H1B2 } \\
\text { representative ITS } \\
\text { sequence }\end{array}$ & $\begin{array}{l}\text { GGAAGTAAAAGTCGTAACAAGGTTTCCGTAGGTGAACCTGCGGAAGGATCATTAATAAATTTTTTTTTGAAAT } \\
\text { AAAAAAAAAAATAATAATCATCCTACCCTTTGTGAATTTGTTTTGTTGAATAATTTTTTAAAATTATTTATTGTC } \\
\text { TATCCAAGTTGGAAAGTTGTAAAAGACTCGAAGACTTGGAGCGGCATAAATAAATTTAAAGACTTTTTTCTAA } \\
\text { AATTAAACTTTTTGTATTCATTTGTCTAAAATAATTTTATAATTTATAAAAACAACTTTGACAATGGATCTCTT } \\
\text { GGTTCTCGCAACGATGAAGAACGCAGCAAAATGCGATAAGTAATGTGAATTGCAGAATACGTGAATCATCGA } \\
\text { ATCTTCGAACGCATATTGCACTTTTTTAGTTTACTAAAAAAGTATGTCTGTTTGAGTATCAGTAAAATATTCTCA } \\
\text { TAAAATTTTTAATTTTATGGTTATGAGTGTTTTACAACGATAAACAAGTTGTTTCACTTTAAATTGAATTTTTGA } \\
\text { AGAAATTCTTTAATTCAAATTAAATTTGGATAAAATTTTCCCGTAAAAAGGAATAACTATACAAGTTAAAAAG } \\
\text { AATTTGGATTTCTTTTATAAAAGGAAATTTATTAAATAACTTGATCTCAAATCAGATAAGAGTACCCGCTGAC } \\
\text { TTAAGCATATTAATAAGCGGAGGA }\end{array}$ \\
\hline $\begin{array}{l}\text { Illumina sequencing } 16 \mathrm{~S} \\
\text { amplicon primers }\end{array}$ & $\begin{array}{l}\text { P5-783F: tcgtcggcagcgtcagatgtgtataagagacagGGWTTAGAWACCCBDGTAGTCC } \\
\text { P7-894R: gtctcgtgggctcggagatgtgtataagagacagCGTACTYCCCAGGYGG }\end{array}$ \\
\hline $\begin{array}{l}\text { Illumina sequencing ITS } \\
\text { amplicon primers }\end{array}$ & $\begin{array}{l}\text { P5-ITS1: tcgtcggcagcgtcagatgtgtataagagacagTCCTACCCTTTGTGAATTTG } \\
\text { P7-ITS4: gtctcgtgggctcggagatgtgtataagagacagCTGCGTTCTTCATCGTTGCG }\end{array}$ \\
\hline
\end{tabular}


648 Table S2: Metagenomic sequencing statistics

\begin{tabular}{|l|l|l|l|l|}
\hline Assembly & $\begin{array}{l}\text { Whole } \\
\text { Metagenome }\end{array}$ & $\begin{array}{l}\text { Methanosphaera } \\
\text { Bin }\end{array}$ & $\begin{array}{l}\text { Methanocorpusculum } \\
\text { Bin }\end{array}$ & $\begin{array}{l}\text { Sphaerochaeta } \\
\text { Bin }\end{array}$ \\
\hline $\begin{array}{l}\text { \# contigs }(>500 \\
\text { bp) }\end{array}$ & 203 & 27 & 67 & 65 \\
\hline $\begin{array}{l}\text { Total Length } \\
\text { (bp) }\end{array}$ & 6659799 & 1779166 & 2045527 & 2880050 \\
\hline GC \% & 48.26 & 29.68 & 53.35 & 55.74 \\
\hline N50 & 28 & 4 & 10 & 14 \\
\hline L50 $(\mathrm{bp})$ & 74369 & 128533 & 75586 & 77038 \\
\hline
\end{tabular}

649

650 Figure S1: Methane Production over time

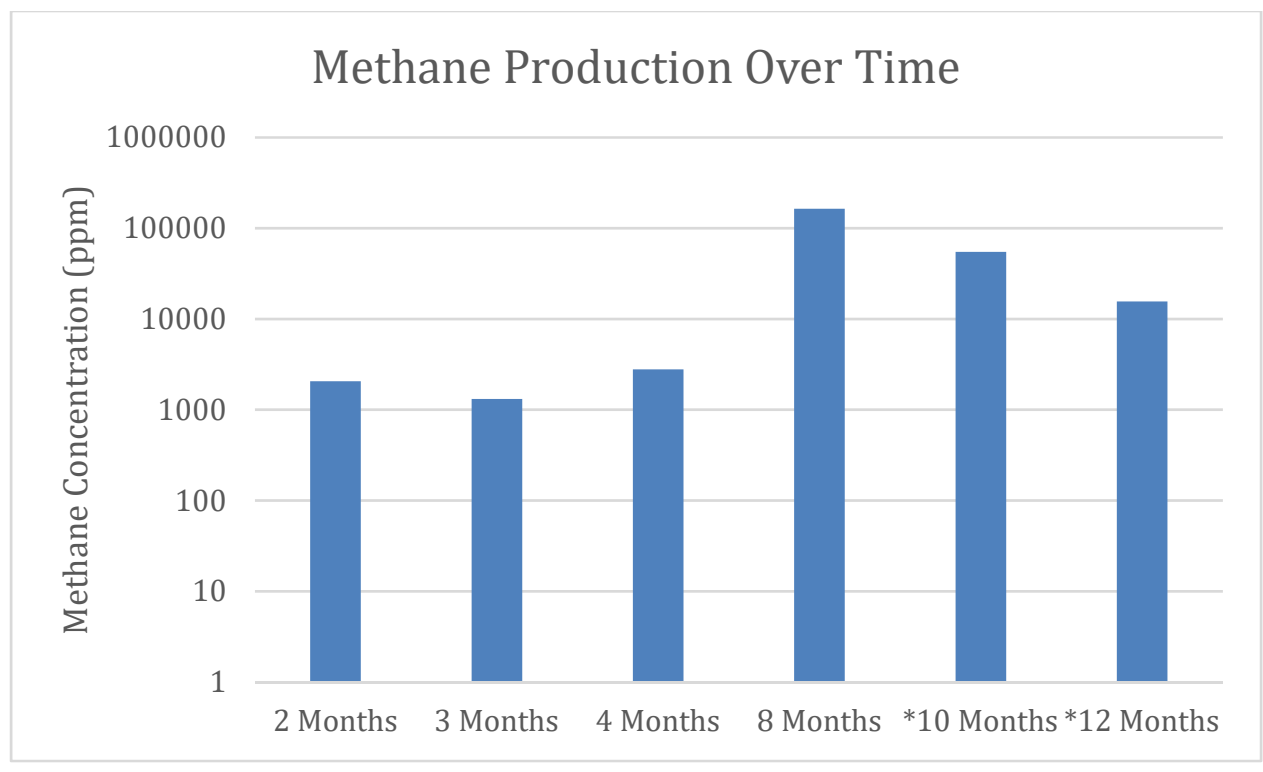

652 Figure S2: Phylogeny of Consortia Members 
A

Tree scale: 0.1
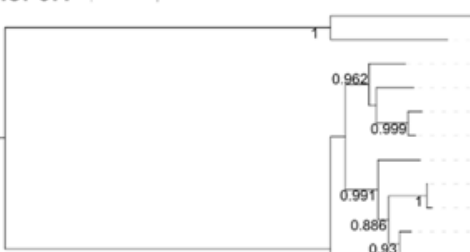

AY997060.1 Monoblepharella sp. M15

AY997061.1 Monoblepharella mexicana isolate AFTOL-ID 33

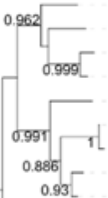

KX889632.1 Pinomyces sp. isolate CaDo16e

KY 399730.1 Piromyces finnis

KY368103.1 Piromyces sp. strain CN4

JF974108.1 Piromyces sp. I GRL-10

JF974109. 1 Caecomyces sp. A GRL-11

EU043229.1 Cyllamyces sp. CB3B1 18S ribosomal RNA gene

FJ501277.1 Cyllamyces sp. AF-CTS-CHCy1

AY997042.1 Cyllamyces aberensis isolate AFTOL-ID 846

0.93__ F.J483845.1 Cyllamyces aberensis isolate EO17

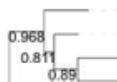

AF 1701871 Anaeromecos sp. JB-1999

NR 148182.1 Anaeromyces robustus

$\left[\begin{array}{l}\text { A.J864475.1 Orpinomyces sp. OUS1 } \\ \text { JQ782553.1 Orpinomyces sp. OYR2 }\end{array}\right.$

$\mathrm{H} 1824$

$T_{\text {b.802 }}{ }^{H 1822}$

0.9 H182

$0.81 . \quad K C 431216.1$ Uncultured Neocallimastigales clone $238 S 18$ internal transcribed spacer 1

B

Tree scale: 0.1

653

654 Figure S3: Morphological features of Piromyces sp. H1B2
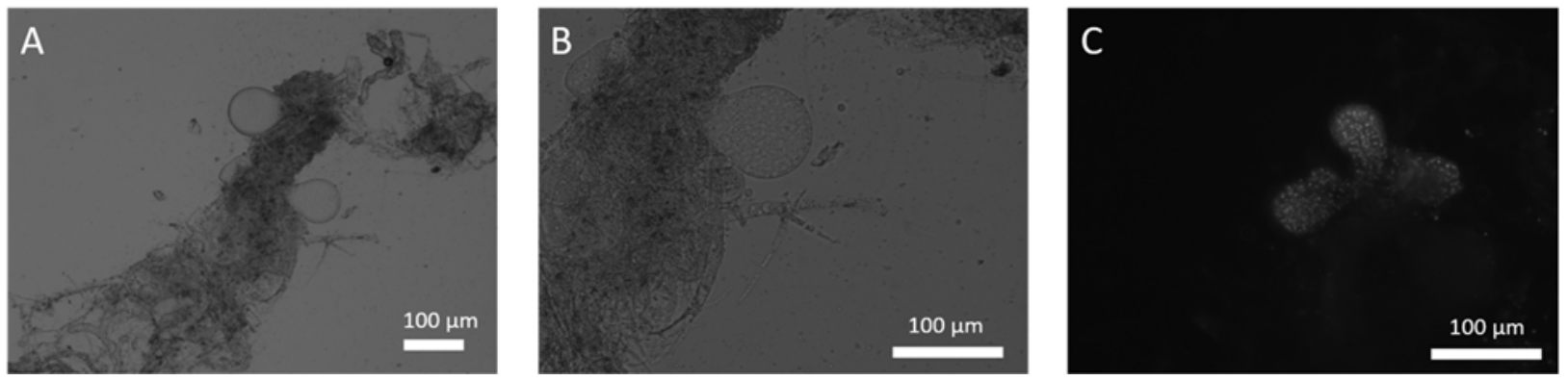

656 Figure S4: Genome Completion as Determined by CheckM 
bin. 1

bin. 3

bin. 2

Single-copy

1
Missing

0
Heterogeneity

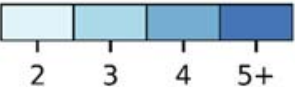

Contamination

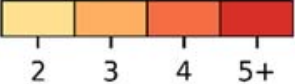

658 


\section{Supporting Information: Top-down Enrichment Guides in Formation of Synthetic Microbial Consortia for Biomass Degradation}

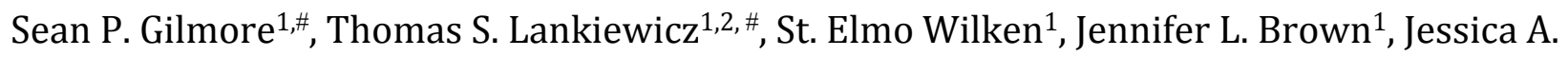

Sexton ${ }^{1}$, John K. Henske ${ }^{1}$, Michael K. Theodorou³, David L. Valentine ${ }^{4}$ and Michelle A. $0^{\prime}$ Malley $^{1^{*}}$

${ }^{1}$ Department of Chemical Engineering, University of California, Santa Barbara, Santa Barbara, CA, 93106, United States

${ }^{2}$ Department of Ecology, Evolution, and Marine Biology, University of California, Santa Barbara, Santa Barbara, CA, 93106, United States

${ }^{3}$ Harper Adams University, Agriculture Centre for Sustainable Energy Systems, Newport, Shropshire, TF10 8NB, United Kingdom

${ }^{4}$ Department of Earth Science and Marine Science Institute, University of California, Santa Barbara, Santa Barbara, CA, 93106, United States

${ }^{*}$ Corresponding Author

\# Equal Contributions 
Discussion S1: Meta-analysis of Published Literature Related to Methanogen-Fungus CoCultivation

Metabolite exchanges occur between methanogenic archaea and anaerobic fungi in our datasets and others (cited in Table S4 below). Many previously published studies suggest that fungus-methanogen interactions accelerates the deconstruction of plant biomass, resulting in increased fermentation gas production. To address this hypothesis, we analyzed

literature purporting to demonstrate increased substrate consumption in co-cultures of anaerobic fungi and methanogens relative to fungal monocultures, and investigated the data from these studies to determine whether suggested outcomes were statistically significant (Bauchop and Mountfort 1981; Mountfort et al. 1982; Marvin-Sikkema et al. 1990; Teunissen et al. 1991, 1992). Overall, we found that previous reports of increased deconstruction by co-culture were limited to qualitative assessments of substrate degradation rates.

Data were extracted from previous publications that concluded the presence of methanogenic archaea increased substrate deconstruction by anaerobic fungi and tested for statistical differences in rate of cellulose deconstruction using analysis of co-variance (ANCOVA) in the same manner that we examined our own deconstruction experiments presented here (Table S4). Contrary to concluding statements made in the publications themselves, we found no statistically supported evidence that co-cultivation with methanogens increased the rate of substrate deconstruction by these cultures $(\mathrm{p}=0.18-0.99$, Table S3). Given the revised interpretation of the data, results agree that a fungalmethanogen syntrophy does not necessarily increase rates of substrate deconstruction. 
Table S1: Primers used and 16S and ITS sequences from enriched consortia

\begin{tabular}{|c|c|}
\hline $\begin{array}{l}\text { Sanger Sequencing } \\
\text { Methanogen-specific } \\
16 S \text { primers }\end{array}$ & $\begin{array}{l}\text { Met86F: GCTCAGTAACACGTGG } \\
\text { Met1340R: CGGTGTGTGCAAGGAG }\end{array}$ \\
\hline $\begin{array}{l}\text { Methanosphaera sp } \\
\text { H1B2 Sanger 16S } \\
\text { sequence }\end{array}$ & $\begin{array}{l}\text { GCTCAGTAACACGTGGATAACTTACCCTTAGGACTGGGATAACTCTGGGAAACTGGGGATAATACCGGATAT } \\
\text { TAGGCTATGCCTGGAATGGTTGGCTTGAAACGTATTTTCGCCTAAGGATAGATCTGCGGCTGATTAGGTC } \\
\text { GTTGGTGGGGTAATGGCCCACCAAGCCTATGATCGGTACGGGTTGTGAGAGCAAGAGCCCGGAGATGGAAC } \\
\text { CTGAGACAAGGTTCCAGACCCTACGGGGTGCAGCAGGCGCGAAACCTCCACAATGTACGAAAGTGCGATGG } \\
\text { GGGAATCCCAAGTGTTATTCTTAACAGAATAGCTTTTCATTAGTGTAAAAAGCTTTTAGAATAAGAGCTGGGC } \\
\text { AAGACCGGTGCCAGCCGCCGCGGTAACACCGGCAGCTCGAGTGGTAGCTGTTTTATTGGGCCTAAAGCGTT } \\
\text { CGTAGCCGGTTTGATTAGTCTTTGGTGAAAGCTTGTAGCTTAACTATAAGAATTGCTGGAGATACTATCAGAC } \\
\text { TTGAAGTCGGGAGAGGTTAGAGGTACTACCGGGGTAGGGGTGAAATCCTATAATCCTGGGAGGACCACCTG } \\
\text { TGGCGAAAGCGTCTAACTAGAACGATCTTGACGGTGAGTAACGAAAGCCAGGGGCGCGAACCGGATTAGAT } \\
\text { ACCCGGGTAGTCCTGGCCGTAAACGATGTGGACTTGGTGTTGGAATGGCTTCGAGTTGTTCCAGTGCCGAAG } \\
\text { GGAAGCTGTTAAGTCCACCGCCTGGGAAGTACGGTCGCAAGACTGAAACTTAAAGGAATTGGCGGGGGAGC } \\
\text { ACCACAACGCGTGGAGCCTGCGGTTTAATTGGATTCAACGCCGGACATCTCACCAGGAGCGACAGCAGAATG } \\
\text { ATAATCAGGTTGATGACCTTATTTGACTAGCTGAGAGGAGGTGCATGGCCGCCGTCAGCTCGTACTGTGAAAG } \\
\text { CGTCCTGTTAAGTCAGGCAACGAGCGAGACCCACGCCCTTAGTTACCAGCTTAATCTTCGGATTGATGGGCAC } \\
\text { ACTAAGGGGACCGCCAGTGATAAACTGGAGGAAGGAGTGGACGACGGTAGGTCCGTATGCCCCGAATCTCC } \\
\text { TGGGCTACACGCGGGCTACAATGGCTATAGCAATGGGTTTCTTCACTGAAAAGTGGGATAATCTCCTAAATA } \\
\text { TAGTCTTAGTTCGGATTGAGGGCTGTAACTCGCCCTCATGAAGCTGGAATGCGTAGTAATCGCATGTCACAAC } \\
\text { CGTGCGGTGAATACGTCCCTGCTCCTTGCACACACCCG }\end{array}$ \\
\hline $\begin{array}{l}\text { Methanocorpusculum } \\
\text { sp H1B2 Sanger 16S } \\
\text { sequence }\end{array}$ & $\begin{array}{l}\text { GCTCAGTAACACGTGGTTAATCTGCCCTTGGGTGGAGGATACTCCCGGGAAACTGGGGCTAATACTCCATAG } \\
\text { TGAATGCATGCTGGAATGCTGCATTCTCGAAAGATTCATCGCCCAAGGATGAGACTGCGTCCGATTAGGTCG } \\
\text { TTGGTGGGGTAACGGCCCACCAAGCCTTTTATCGGTACGGGTTGTGGGAGCAAGATCCCGGAGATGGATTCT } \\
\text { GAGACATGAATCCAGGCCCTACGGGGCGCAGCAGGCGCGCAAACTTTACAATGCGAGCAATCGTGATAAGG } \\
\text { AAACCCTGAGTGCCTGTCGATGCAGGCTGTTCATATGTTTAAATTGCATGTGAAGAAAGGGCAGGGCAAGAC } \\
\text { CGGTGCCAGCCGCCGCGGTAATACCGGCTGCTCGAGTGATGGCCACTATTACTGGGTTAAAGCGTCCGTAG } \\
\text { CTTGACTGTTAGGTCTCTTGGGAAATCTTTTGGCTTAACCAAAAGGCGTCTAAGAGATACCGGCATCTTGGA } \\
\text { ACTGGGAGAGGTAAGCCGTACTTCGGGGGTAGGAGTGAAATCTTGTAATCCTCGAGGGACGACCTATGGCG } \\
\text { AAGGCAGCTTACCAGAACAGCTTCGACAGTGAGGGACGAAAGCTGGGGAGCAAACGGGATTAGATACCCC } \\
\text { GGTAGTCCCAGCCGTAAACAATGTGCGTTAGGTGTGTCGGTTACCACGCGTAACTGATGCGCCGAAGAGAAA } \\
\text { TCGTGAAACGCACCACCTGGGAAGTACGGTCGCAAGGCTGAAACTTAAAGGAATTGGCGGGGGAGCACCAC } \\
\text { AACAGGTGGAGCCTGCGGTTTAATTGGATTCAACGCCGGACATCTCACCGGATAAGACAGCTGAATGATTGT } \\
\text { CAATCTGAAGGTTTACATGACTAGCTGAGAGGAGGTGCATGGCCGTCGTCAGTTCGTACTGTGAAGCATCC } \\
\text { TGTTAAGTCAGGCAACGAGCGAGACCCACGCCAACAATTGCCAGCAGCATCTCCGGATGGCTGGGGACATTG } \\
\text { TTGGGACCGCCTCTGCTAAAGGGGAGGAAGGAATGGGCAACGGAGGTCAGCATGCCCCGAATTATCCGGG } \\
\text { CTACACGCGGGCTACAATGGCCGGGCAATGGGTAAGGACACCGAAAGGTCAGTCAATCTCCTAACCCCGG } \\
\text { CCTTAGTTAGGATTGCGGGTTGCAACTCACCCGCATGATCTGGAATCTGTAGTAATCGCGTTCACTATAGC } \\
\text { GCGGTGAATACGTCCCTGCTCCTTGCACACACCG }\end{array}$ \\
\hline $\begin{array}{l}\text { Sanger Sequencing ITS } \\
\text { region primers }\end{array}$ & $\begin{array}{l}\text { JB205: GGAAGTAAAAGTCGTAACAAGG } \\
\text { JB206: TCCTCCGCTTATTAATATGC }\end{array}$ \\
\hline $\begin{array}{l}\text { Piromyces sp H1B2 } \\
\text { representative ITS } \\
\text { sequence }\end{array}$ & $\begin{array}{l}\text { GGAAGTAAAAGTCGTAACAAGGTTTCCGTAGGTGAACCTGCGGAAGGATCATTAATAAATTTTTTTTTGAAAT } \\
\text { AAAAAAAAAAATAATAATCATCCTACCCTTTGTGAATTTGTTTGGTTGAATAATTTTTTAAAATTATTTATTGTC } \\
\text { TATCCAAGTTGGAAAGTTGTAAAAGACTCGAAGACTTGGAGCGGCATAAATAAATTTAAAGACTTTTTCTAA } \\
\text { AATTAAACTTTTTGTATTCATTTGTCTAAAATAATTTTTATAATTTATAAAAACAACTTTGACAATGGATCTCTT } \\
\text { GGTTCTCGCAACGATGAAGAACGCAGCAAAATGCGATAAGTAATGTGAATTGCAGAATACGTGAATCATCGA } \\
\text { ATCTTCGAACGCATATTGCACTTTTTTAGTTTACTAAAAAAGTATGTCTGTTTGAGTATCAGTAAAATATTCTCA } \\
\text { TAAAATTTTTAATTTTATGGTTATGAGTGTTTTACAACGATAAACAAGTTGTTTCACTTTAAATTGAATTTTGGA } \\
\text { AGAAATTCTTTAATTCAAATTAAATTTGGATAAAATTTCCCGTAAAAAGGAATAACTATACAAGTTAAAAAG } \\
\text { AATTTGGATTTCTTTTATAAAAGGAAATTTATTAAATAACTTGATCTCAAATCAGATAAGAGTACCCGCTGAC } \\
\text { TTAAGCATATTAATAAGCGGAGGA }\end{array}$ \\
\hline $\begin{array}{l}\text { Illumina sequencing } 16 \mathrm{~S} \\
\text { amplicon primers }\end{array}$ & $\begin{array}{l}\text { P5-783F: tcgtcggcagcgtcagatgtgtataagagacagGGWTTAGAWACCCBDGTAGTCC } \\
\text { P7-894R: gtctcgtgggctcggagatgtgtataagagacagCGTACTYCCCAGGYGG }\end{array}$ \\
\hline $\begin{array}{l}\text { Illumina sequencing ITS } \\
\text { amplicon primers }\end{array}$ & $\begin{array}{l}\text { P5-ITS1: tcgtcggcagcgtcagatgtgtataagagacagTCCTACCCTTTGTGAATTTG } \\
\text { P7-ITS4: gtctcgtgggctcggagatgtgtataagagacagCTGCGTTCTTCATCGTTGCG }\end{array}$ \\
\hline
\end{tabular}


Table S2: Metagenomic sequencing statistics of the prokaryotic strains sequenced from a naturally-enriched microbial consortium.

\begin{tabular}{|l|l|l|l|l|}
\hline Assembly & $\begin{array}{l}\text { Whole } \\
\text { Metagenome }\end{array}$ & $\begin{array}{l}\text { Methanosphaera } \\
\text { Bin }\end{array}$ & $\begin{array}{l}\text { Methanocorpusculum } \\
\text { Bin }\end{array}$ & $\begin{array}{l}\text { Sphaerochaeta } \\
\text { Bin }\end{array}$ \\
\hline $\begin{array}{l}\text { \# contigs } \\
\text { (>500 bp) }\end{array}$ & 203 & 27 & 67 & 65 \\
\hline $\begin{array}{l}\text { Total Length } \\
\text { (bp) }\end{array}$ & 6659799 & 1779166 & 2045527 & 2880050 \\
\hline GC \% & 48.26 & 29.68 & 53.35 & 55.74 \\
\hline N50 & 28 & 4 & 10 & 14 \\
\hline L50 (bp) & 74369 & 128533 & 75586 & 77038 \\
\hline
\end{tabular}


Table S3: Supernatant metabolites measured after 10 days of fungal or co-culture growth on two representative substrates. The top panel depicts metabolites following growth on Whatman filter paper (WP), and the bottom panel shows the same data for growth supported on reed canary grass (RCG). Reported errors represent standard deviations of biological replicates $(n=3)$.

\begin{tabular}{|c|c|c|c|c|c|c|c|}
\hline \multicolumn{8}{|l|}{ WP } \\
\hline & Pressure (psig) & Hydrogen (\%) & Methane (\%) & Reducing sugars (g/L) & Formate $(\mathrm{g} / \mathrm{L})$ & Acetate $(\mathrm{g} / \mathrm{L})$ & Lactate $(\mathrm{g} / \mathrm{L})$ \\
\hline N. californiae & $10.2 \pm 0.4$ & $14.6 \pm 2.6$ & $0.0 \pm 0.0$ & $6.7 \pm 2.9$ & $0.9 \pm 0.0$ & $0.8 \pm 0.1$ & $0.8 \pm 0.1$ \\
\hline N.c. + M. bryantii & $6.2 \pm 0.3$ & $0.0 \pm 0.0$ & $4.1 \pm 0.1$ & $5.6 \pm 0.7$ & $0.0 \pm 0.0$ & $1.0 \pm 0.2$ & $0.1 \pm 0.0$ \\
\hline A. robustus & $4.5 \pm 0.4$ & $8.8 \pm 0.7$ & $0.0 \pm 0.0$ & $0.1 \pm 0.1$ & $0.5 \pm 0.0$ & $2.5 \pm 0.3$ & $0.0 \pm 0.0$ \\
\hline A.r. + M. bryantii & $6.6 \pm 0.4$ & $0.0 \pm 0.0$ & $12.0 \pm 1.7$ & $0.0 \pm 0.0$ & $0.0 \pm 0.0$ & $1.2 \pm 0.2$ & $0.0 \pm 0.0$ \\
\hline \multicolumn{8}{|l|}{ RCG } \\
\hline & Pressure (psig) & Hydrogen (\%) & Methane (\%) & Reducing sugars (g/L) & Formate $(\mathrm{g} / \mathrm{L})$ & Acetate $(\mathrm{g} / \mathrm{L})$ & $\underline{\text { Lactate }(\mathrm{g} / \mathrm{L})}$ \\
\hline N. californiae & $10.4 \pm 0.7$ & $13.6 \pm 2.1$ & $0.0 \pm 0.0$ & $0.5 \pm 0.0$ & $0.7 \pm 0.1$ & $1.0 \pm 0.2$ & $1.0 \pm 0.2$ \\
\hline N.c. + M. bryantii & $10.0 \pm 0.2$ & $0.0 \pm 0.0$ & $4.3 \pm 0.2$ & $0.6 \pm 0.0$ & $0.0 \pm 0.0$ & $1.5 \pm 0.1$ & $0.5 \pm 0.2$ \\
\hline A. robustus & $6.7 \pm 0.1$ & $13.3 \pm 2.4$ & $0.0 \pm 0.0$ & $0.0 \pm 0.0$ & $0.7 \pm 0.1$ & $1.0 \pm 0.0$ & $0.0 \pm 0.0$ \\
\hline A.r. + M. bryantii & $7.9 \pm 0.2$ & $0.0 \pm 0.0$ & $12.7 \pm 3.0$ & $0.1 \pm 0.0$ & $0.0 \pm 0.0$ & $1.2 \pm 0.2$ & $0.0 \pm 0.0$ \\
\hline
\end{tabular}


Table S4: Extraction and re-analysis of published literature shows that fungal-methanogen co-cultivation does not result in increased substrate solubilization rate compared to fungal monoculture. The data purporting to show that methanogens increase the rate of substrate solubilization was analyzed using the same ANCOVA analysis as was used for the experimental data depicted in Figure 5. In each case, it was found that the rate of substrate solubilization difference between fungal monocultures co-cultures was not statistically significant $(p>0.05)$. This finding is in agreement with the results presented in Figure 5 in the main text between $N . c$. and M. b., and A. $r$. and M. $b$., that also found no significant change.

\begin{tabular}{|c|c|c|c|}
\hline$\underline{\text { Source Citation(s) }}$ & $\underline{\text { Organisms }}$ & ANCOVA p-value(s) & $\underline{\text { Notes }}$ \\
\hline The Rumen and its Microbes, Hungate 1966 & N/A & $\mathrm{N} / \mathrm{A}$ & Origin of hypothesis \\
\hline $\begin{array}{l}\text { Bauchop and Mountfort Applied and Environmental } \\
\text { Microbiology, } 1981\end{array}$ & $\begin{array}{l}\text { Unidentified fungus } \\
+ \text { M. barkeri and Methanobrevibacter sp. RA1 }\end{array}$ & $p=0.1269$ & \\
\hline $\begin{array}{l}\text { Mountfort et al. Applied and Environmental } \\
\text { Microbiology, } 1982\end{array}$ & Organisms not identified & $\mathrm{p}=0.9155$ & \\
\hline $\begin{array}{l}\text { Marvin-Sikkema et al. Applied and Environmental } \\
\text { Microbiology, } 1990\end{array}$ & $\begin{array}{l}\text { Neocallimastix sp. } \mathrm{L} 2 \\
+ \text { M. bryantii, M. smithii, or M. aboriphilus }\end{array}$ & $p=0.1977$ & \\
\hline Teunissen et al. Archives of Microbiology, 1991 \& 1992 & $\begin{array}{l}\text { Several Neocallimastix and Piromyces } \\
+M \text {. formicicium }\end{array}$ & $\begin{array}{c}\mathrm{p}=0.32,0.62,0.18 \\
0.53\end{array}$ & $\begin{array}{l}\text { Data in } 2 \text { separate papers; } 4 \\
\text { different comparisons }\end{array}$ \\
\hline Li et al. 2017 Journal of Basic Microbiology, 2017 & $\begin{array}{l}\text { Piromyces sp. F1 } \\
+ \text { M. thaueri }\end{array}$ & $p=0.99$ & $\begin{array}{c}\text { Author scorrectly note no enhanced } \\
\text { degradation by coculture }\end{array}$ \\
\hline This work & $\begin{array}{l}\text { Neocallimastix californiae } \\
+ \text { M. bryantii }\end{array}$ & $p=0.3554$ & \\
\hline This work & $\begin{array}{l}\text { Neocallimastix sp. S3 } \\
+ \text { M. bryantii }\end{array}$ & $p=0.1844$ & \\
\hline This work & $\begin{array}{l}\text { Anaeromyces robustus } \\
+M . \text { bryantii }\end{array}$ & $\mathrm{p}=0.1831$ & \\
\hline
\end{tabular}




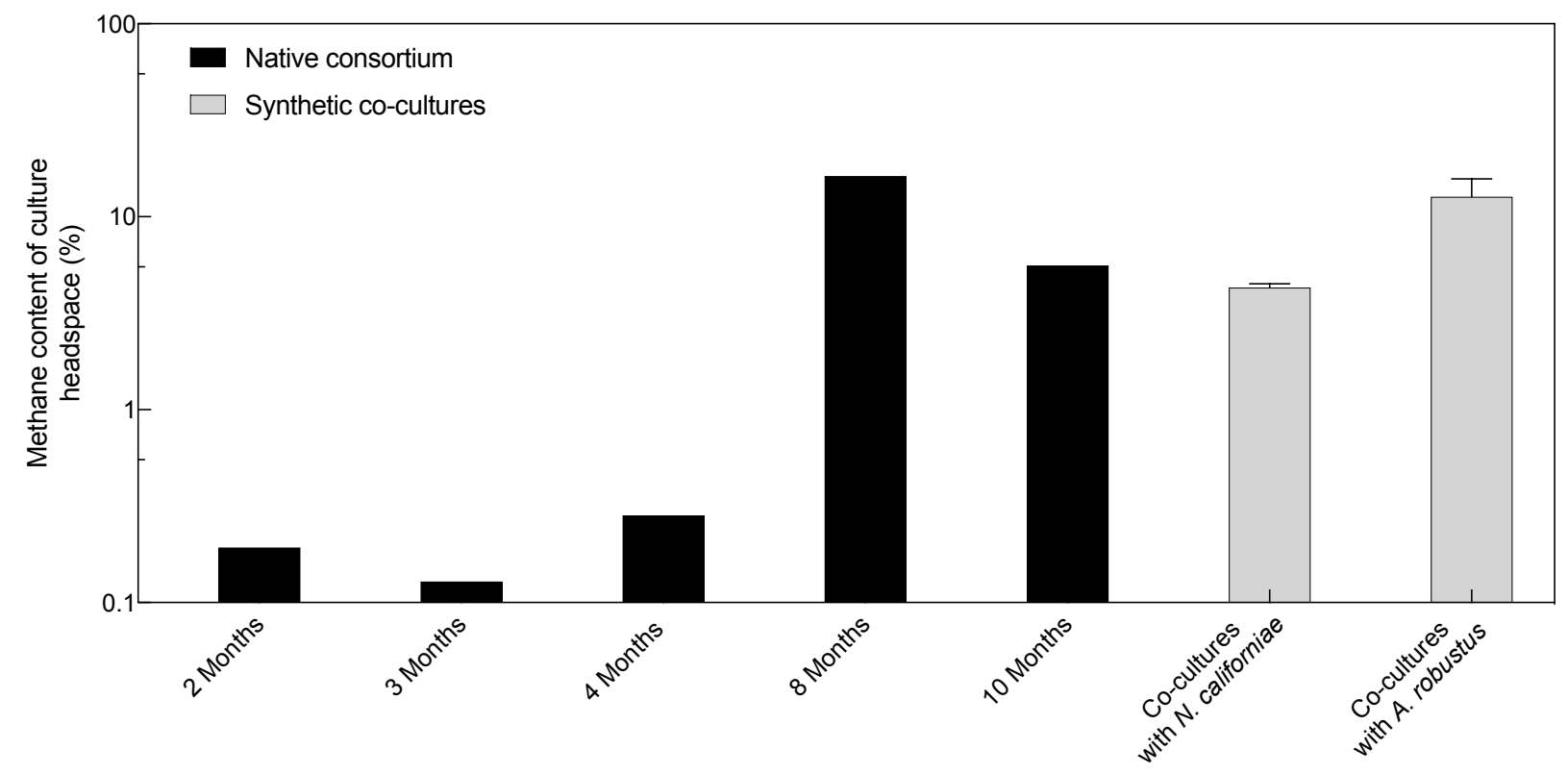

Figure S1: Methane production increases in an enriched native community over time and is comparable to methane produced by synthetic pairings. Headspace methane concentrations were periodically measured via gas chromatography (GC) during consecutive batch culture of the enriched native consortium and during subsequent experiments with indicated synthetic co-cultures. Methane production by the native consortium was low at initial enrichment, but eventually reached the levels seen in synthetic co-culture experiments. For the native consortium, bars represent single measurements of the culture headspace for methane percentage, but for synthetic pairings bars represent the means of biological triplicates and error bars represent the standard deviation. 


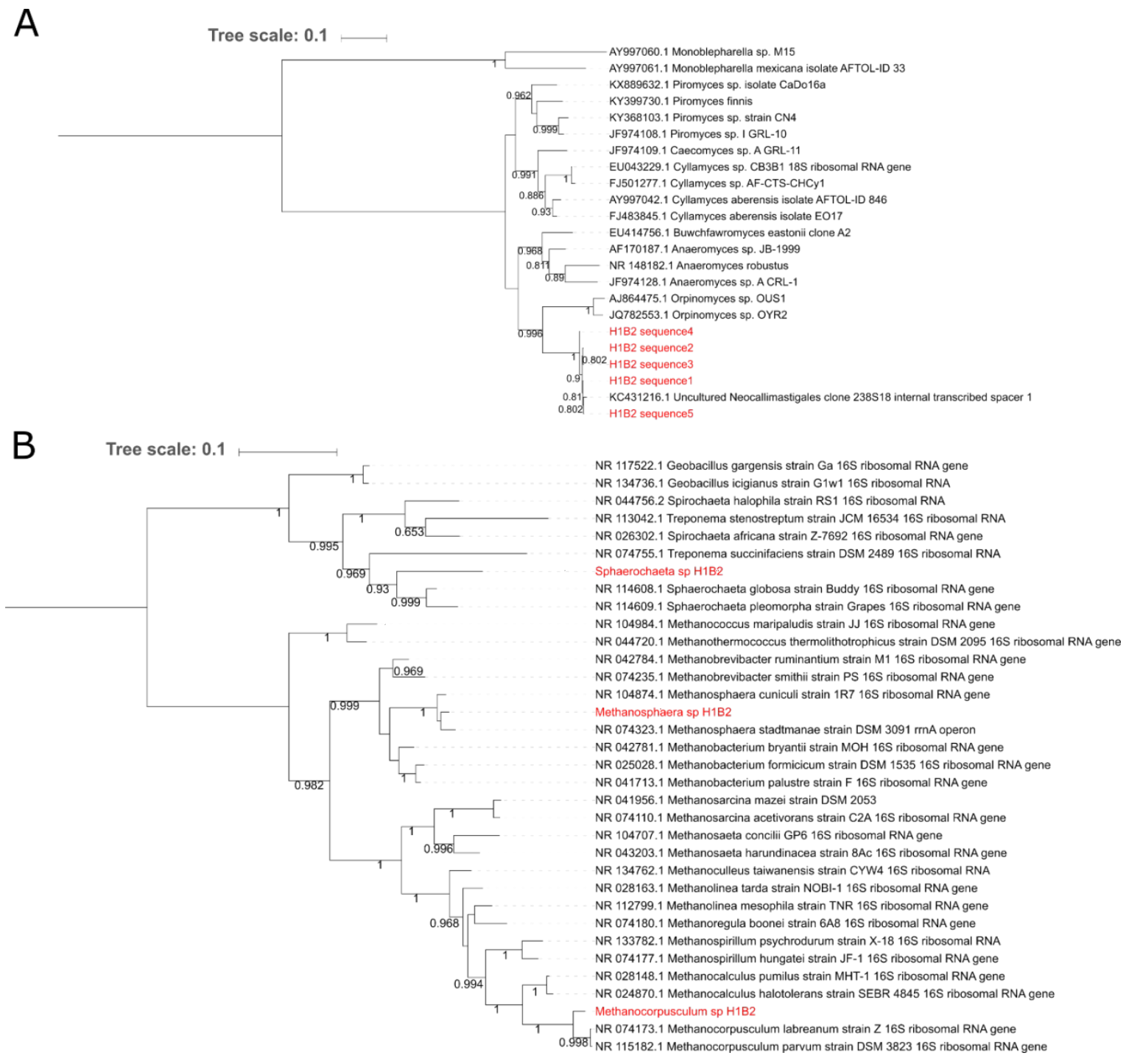

Figure S2: Phylogenetic placement of the microbial consortium members enriched

from horse feces. A) ITS sequence alignment reveals that the fungus (H1B2 sequence 1-5)

clusters distinctly from other sequenced fungal isolates. Five different Sanger sequencing runs were used due to the difficulties associated with the high AT\% in the anaerobic fungal ITS regions B) $16 \mathrm{~S}$ alignment of the three distinct sequences reveals two methanogens (Methanocorpusculum and Methanosphaera, denoted as Methanosphaera sp. H1B2 and Methanocorpusculum sp. H1B2) and one bacterium (Sphaerochaeta, denoted Sphaerochaeta sp. H1B2). 

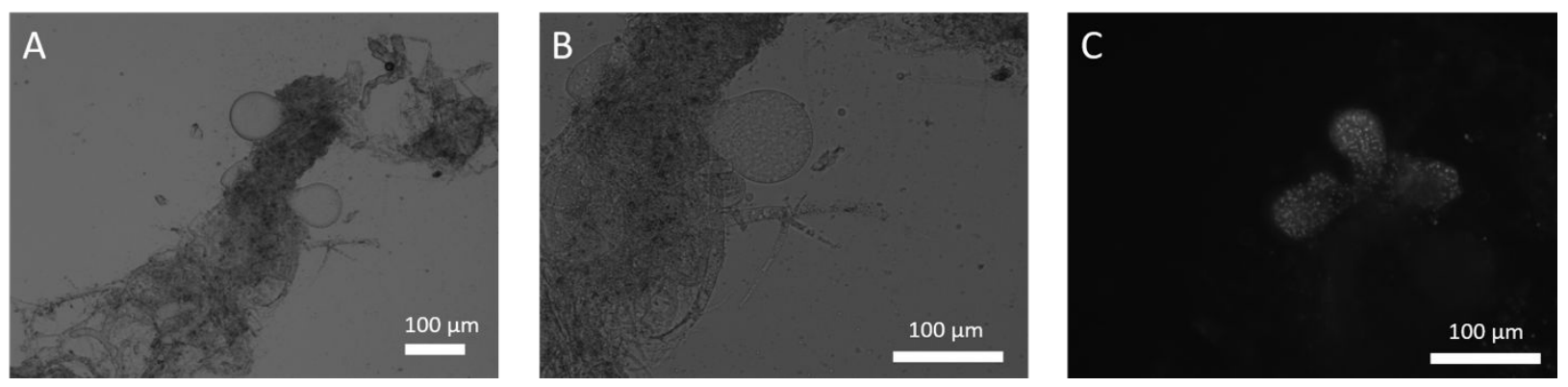

Figure S3: Light microscopy of the top-down enriched anaerobic consortium reveal a

Piromyces-like fungus. Light microscopy of the fungal isolate (A and B) revealed abundant tapering rhizoids, multinucleated sporangia, and absence of nuclei in rhizoids with DAPI staining (C), which are generic characteristics for Piromyces. 


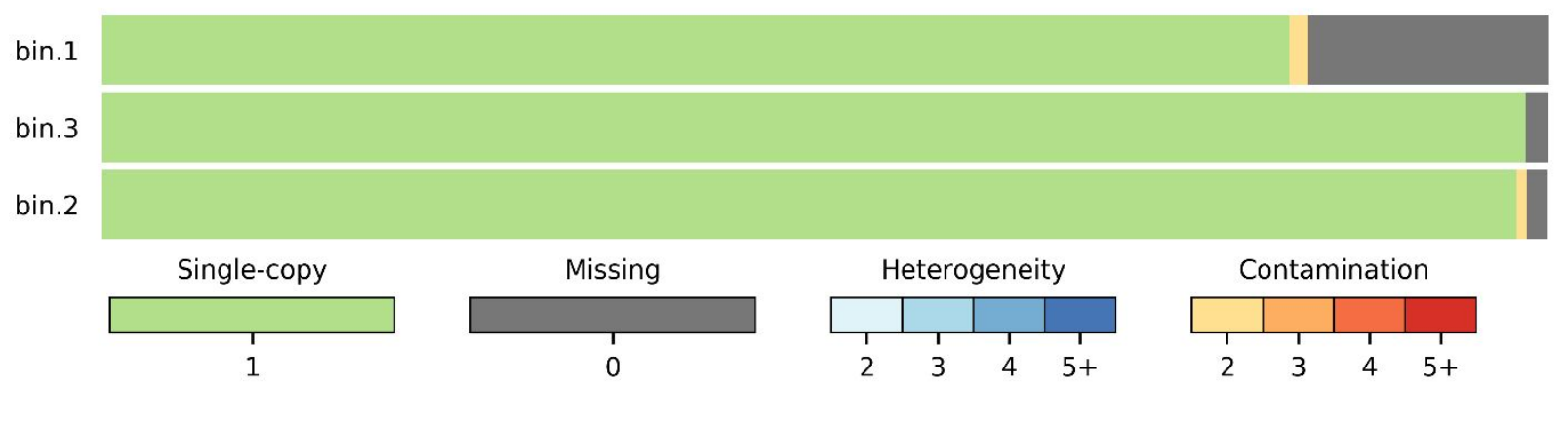

Figure S4: Metagenome assembled genome completion is high and contamination is low as determined by CheckM. The assembled metagenome was binned into distinct genome bins based on read coverage and tetranucleotide frequency using Metabat and CONCOCT. The completeness and quality of each bin was determined using the single copy gene sets employed through the CheckM method. Each bin achieved better than $90 \%$ completion with limited $(<2 \%)$ contamination and heterogeneity, qualifying as high quality Metagenome Assembled Genomes. 


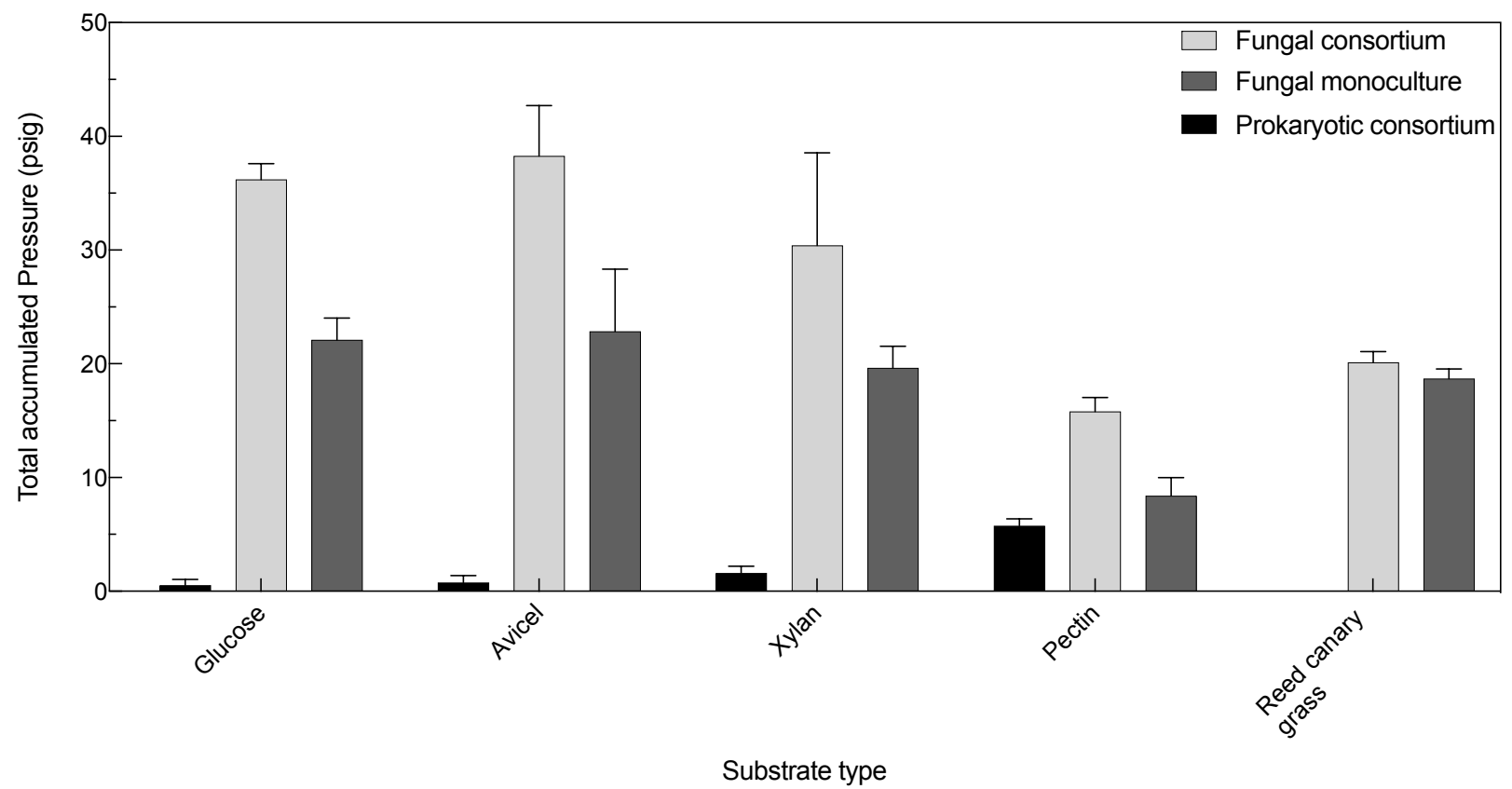

Figure S5: Total fermentation gas production by the enriched natural microbial consortia, the prokaryotic component of the consortium, and the fungal component of the consortium. Accumulation of total fermentation gases were tracked for 10 days in triplicate cultures that were vented daily, supported on the indicated substrates. Pressure accumulated was measured using the pressure transducer technique. Error bars represent the standard error of the mean $(n=3)$. 


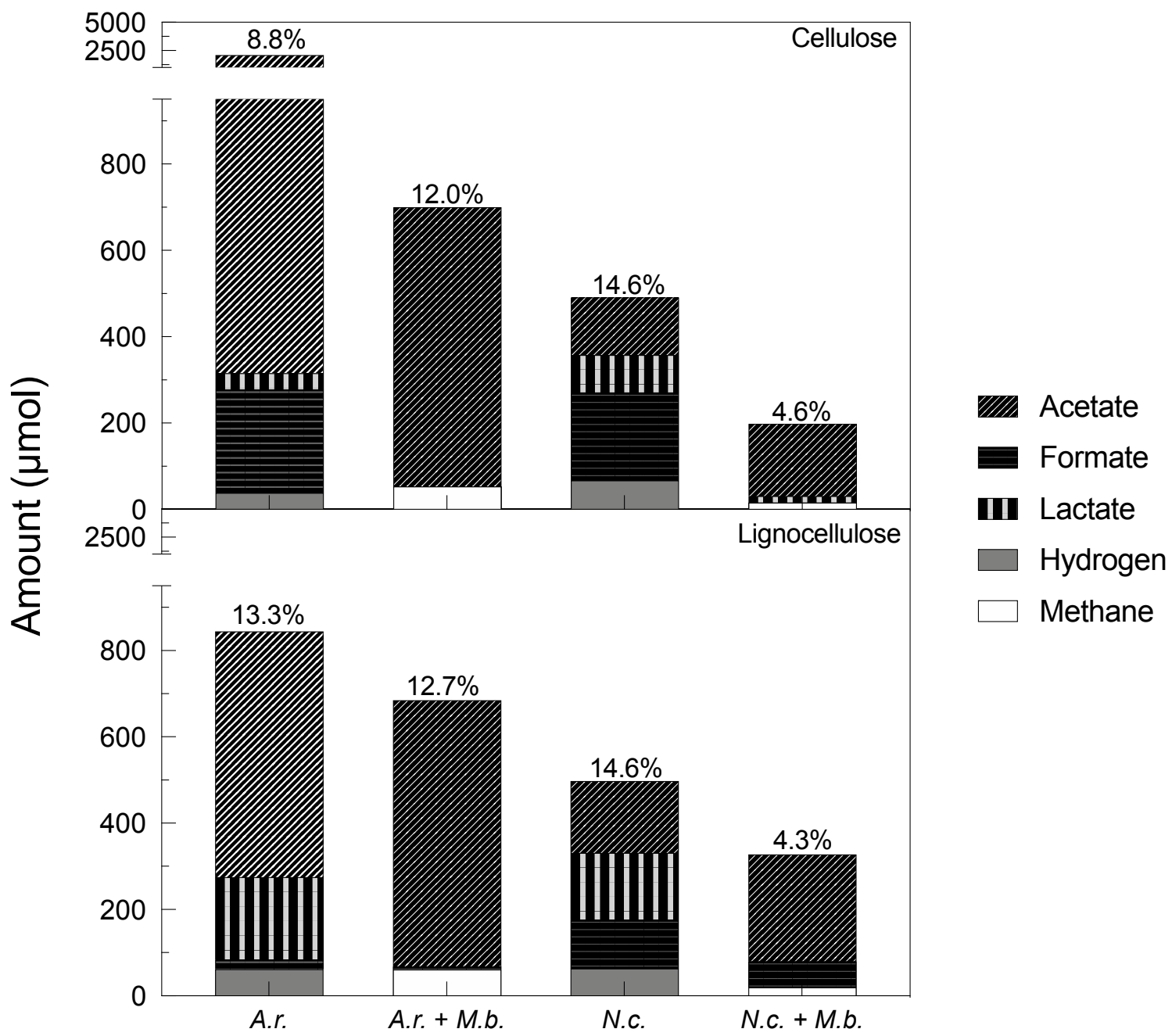

Figure S6: The presence of the methanogenic archaeon Methanobrevibacter bryantii (M.b.) alters the end-point metabolite profile of anaerobic fungi grown on both cellulosic and lignocellulosic substrates. Data were collected for two strains of anaerobic fungi, $N$. californiae (N.c.) and A. robustus (A.r.) in both the presence and absence of the methanogen M. bryantii (M.b.). The bar displayed for each metabolite is the result of averaging three biological replicates. Standard deviations are not shown for clarity; however, values obtained from liquid chromatography have standard deviations less than $10 \%$ of the mean, and values obtained from gas chromatography have standard deviations 
less than $17 \%$ of the mean (Table S3). Percentages indicated at the top of each bar-stack refer to the mean percentage of headspace gas that was measured as hydrogen in monocultures or as methane in co-cultures $(n=3)$. 

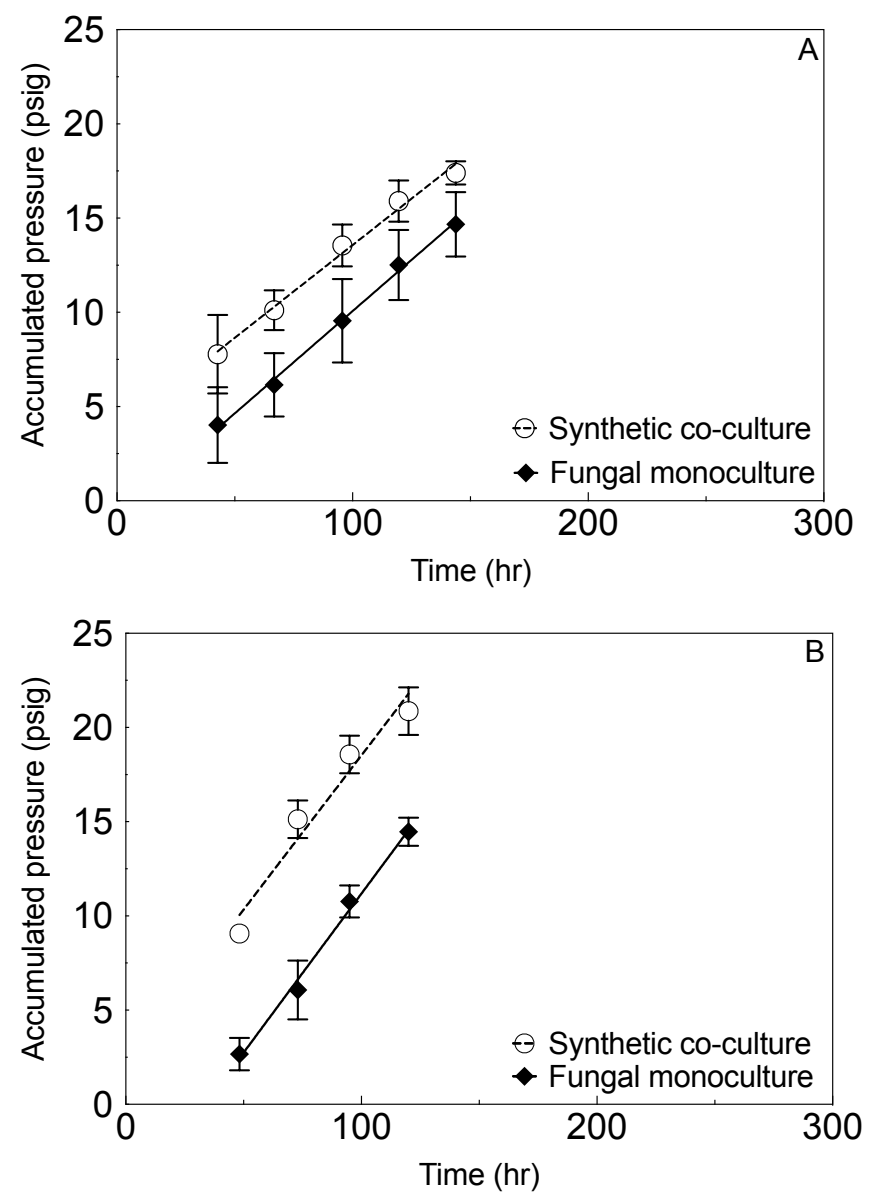

Figure S7: Comparison of gas production rates of fungal monocultures with gas production rates of synthetic co-cultures of anaerobic fungi and methanogens: Two anaerobic fungi were grown in isolation and paired with the methanogen Methanobrevibacter bryantii (M.b.) while total fermentation gas production was tracked using the pressure transducer technique. Data and linear regression models for Neocallimastix californiae (N.c.) and M.b. are shown in panel A while data and regression models for Anaeromyces robustus (A.r.) and M.b. are shown in panel B. Linear regressions were calculated for the steady state portion of culture growth which excluded lag and stationary phases. Slopes of linear regressions (N.c. $=0.11 \pm 0.00 \mathrm{psig} / \mathrm{hr}$, N.c. + M.b. $=0.10 \pm 0.00$ $\mathrm{psig} / \mathrm{hr}, \quad$ A.r. $=0.17 \pm 0.01 \mathrm{psig} / \mathrm{hr}, \quad$ A.r. + M.b. $=0.16 \pm 0.03 \mathrm{psig} / \mathrm{hr}$ ) were compared using 
ANCOVA which determined that there was no statistically significant difference in the rate of fermentation gas production $(\mathrm{p}>0.05)$ 
Supplemental Database 2: Transporter analysis of metagenomes

Number of high quality sugar transporters per MAG by predicted substrate

$v \underline{\text { Predicted substrate }}_{v} \quad$ MAG $=$ Sphaerochaeta_H1B`Methanosphaera_H1B:

Maltose or maltooligosaccaride

5

Mannose

2

Glucose or galactose

2

Lipopolysaccride

Alginic acid

1

Xylan

1

1

$\mathrm{N}$-acetyl glucosamine

0

Glycosyl groups

0

12

1 
Methanocorpusculum_H1B2

0

0

0

0

0

0

0

2

2 